Article

\title{
The Standard Cosmological Model: Basic Geometric and Kinematic Features
}

\author{
Dmitrij Nagirner ${ }^{1,+}{ }^{-}$, and Svetlana Jorstad ${ }^{2, \ddagger 1,+}+(\mathbb{C}$ \\ 1 Astronomy Department, St. Petersburg State University, Universitetskij Pr. 28, Petrodvorets, 198504 St. \\ Petersburg, Russia \\ 2 Boston University, 725 Commonwealth Ave., Boston, MA, 02215, USA \\ * Correspondence: dinagirner@gmail.com
}

\begin{abstract}
We present a brief history of the construction of models of the universe, followed by calculations of quantitative characteristics of basic geometric and kinematic properties of the Standard Cosmological Model $(\Lambda \mathrm{CDM})$. Using the Friedmann equations of uniform space, we derive equations characterizing a $\Lambda \mathrm{CDM}$ model that describes a universe corresponding to current observational data. The equations take into account the effects of radiation and ultra-relativistic neutrinos. It is shown that the universe at very early and late stages can be described to sufficient accuracy by simple formulas. Certain important moments of cosmic evolution are determined: the times when densities of the gravitational components of the universe become equal, when they contribute equally to the gravitational force, when the accelerating expansion of space begins, and several others. The dependences of different distances on redshift and the scale factor of space are derived. The distance to the sphere that expands with the speed of light (the Hubble distance), and its current and future acceleration, are found. Concepts of a horizon, second inflation, and second horizon are discussed. We consider the remote future of the universe and the opportunity, in principle, of connection with extraterrestrial civilizations.
\end{abstract}

Keywords: Standard model, first and second horizons, cosmological constant

\section{Introduction}

We present a brief history of the construction of cosmological models, starting with the models of A. Einstein, V. de Sitter, A. Friedmann, and J. Lemaître, and describe observational attempts to choose model parameters such as the Hubble constant and the sign of curvature of space-time. We follow the evolution of understanding of the role of the cosmological constant from its (1) introduction by Einstein to compensate for gravitational attraction, (2) later near-elimination from the theory, (3) association with some substance, and (4) recognition of this substance as the main component of the universe, currently named "dark energy," that defines the acceleration of the expansion of space. All of these, along with ongoing technological progress, have led to the formulation of the so-called "Standard" model, which is the model that most adequately describes the existing universe.

We use the metric of Friedmann-Robertson-Walker and equations of Friedmann-Lemaître, which form the foundation of the majority of the models. We recall the definition of the "critical density" and discuss the densities of four noninteracting components of the universe: dark energy, dust matter, which includes baryonic and dark matter, radiation, and ultrarelativistic neutrinos. We derive the laws of evolution of the density of these components. Inclusion of these components allow to obtain the general solution of the equations while highlighting the case of flat space-time.

We consider the problem of the propagation of radiation in the universe from a source to the observer, a term of the geometrical horizon that arises in this case, and discuss different concepts of distances in the universe. The relationship between a time derivative of the metric distance, interpreted as the speed of expansion, and the distance itself — the Hubble-Lemaittre law — and redshift is given. We explain why the cosmological redshift is not similar to the classic Doppler effect. 
We adopt the modern parameters of the Standard model: the temperature of the cosmic microwave background $(\mathrm{CMB})$, the temperature of neutrino gas associated with the $\mathrm{CMB}$, the Hubble constant, and a share of dark energy in the total density. These parameters allow us to calculate analytically the evolution of the mass density of the four components and their relative contributions to the critical density, which are employed to specify relations between scale characteristics of the model and time. We derive approximate formulas of these relations at the initial and remote-future epochs of expansion. The evolution of a role of the components at different epochs is analysed and values of distances, speeds, and accelerations as functions of redshift are calculated. We estimate a duration of the time interval needed to detect a change of the redshift and apparent luminosity of a remote object with time.

The reasons for the existence of a second inflation and a second horizon are explained. Distances to the horizons and to the place where the rate of expansion is equal to the speed of light (the Hubble distance) are determined along with their speeds and accelerations. Finally, we discuss whether a connection with extraterrestrial civilizations can be established in principle.

Based on the Standard model, the anisotropy of the cosmic microwave background, primary nucleosynthesis, and the formation of large-scale structures in the universe are reproduced quite accurately, as is presented in well known monographs, for example, [1-5]. Here we provide an overview of the geometric and kinematic properties of the modern Standard cosmological model.

\section{Stages of building a model}

The path to the construction of a cosmological model, now known as the Standard model or $\Lambda \mathrm{CDM}$, was quite long. The history of this path is described in many cosmology textbooks. Here we summarize the main stages of this history.

The intention to build a cosmological model, that is, a model of the entire universe, and not only the solar system or the Galaxy, appeared as soon as A. Einstein (1917) has formulated the equations of the general theory of relativity [7] (1916), which describe gravitational fields and the behavior of matter in them. He applied the equations to the homogeneous and isotropic distribution of matter (following the "cosmological principle") and tried to find a stationary solution of these equations to avoid problems of an origin and initial ultra-dense stages in the history of the universe. To achieve this, he had to supplement the equations with the cosmological term, so-called cosmological constant, or $\Lambda$ parameter [7], which corresponds not to gravitational attraction, but to repulsion. The stationary solution was obtained only for a closed universe and, moreover, as A. Eddington showed [8], turned out to be unstable.

Another stationary solution was obtained by de Sitter [9] (1917) also for a closed universe, but not containing matter (empty space). In this model, the passage of time at a certain point of space depends on the distance to this position from the observer. The redshift of the spectrum of a remote object was explained by the fact that a clock around it was running more slowly than that of an observer on the Earth. According to this theory, the radius of the curvature of space that does not change with time, $R=R_{0}$, is directly connected with the cosmological constant: $\Lambda=3 / R_{0}^{2}$. This model has prompted numerous works on the study of the "de Sitter World" itself, in which properties of the symmetry of space in different coordinates (see the references in [8]) were considered. In fact, these works are more of a mathematical character than of cosmological insight, although they deal with some astronomical aspects and observed values (speeds of galaxies, size of universe, etc.).

The first non-stationary solutions of the Einstein equations were obtained by a Russian mathematician, fluid mechanician, and meteorologist A.A. Friedmann (1888-1925) for a matter without pressure, uniformly distributed in space, first with a positive curvature [10] (1922), then with a negative curvature [11] (1924). In these works he also studied cases with positive and negative values of the cosmological constant.

The Belgian theorist G. Lemaître (1894-1966) also obtained non-stationary solutions [12] in 1927, not knowing about the works by Friedmann (when reprinting the article in English, he mentioned the Friedmann work [10]). Lemaître was the first who included radiation as a component of the universe 
and analytically derived the Hubble law ahead of its observational discovery (now this law is called the Hubble-Lemaitre law). The idea of the Big Bang at the beginning of the expansion of the universe belongs to Lemaître as well [13].

As for the cosmological constant, Einstein first doubted the need for its introduction [14], and completely abandoned it when non-stationary solutions of the equations were obtained [15]. In 1929 E. Hubble, using the 100-inch Mount Wilson Telescope, discovered that the redshift of lines (translated by him into speed) in spectra of weak nebulae (which he had found to be galaxies) increases with the distance to them. He interpreted this as recession. This was considered as a proof of a non-stationarity of the universe. However, the values of the parameters relevant to the universe remained uncertain and had to be determined by observations.

Observers turned to clarification of the cardinal question of whether the universe is closed or open, that is, what is larger: the actual mass density of matter in space, $\rho$, or the critical density, $\rho_{c}$, delimiting closed and open models. At that time it was believed that the universe consists of primarily observable objects that radiate or absorb light, such as planets, stars, galaxies, gas, and dust that form the baryonic component of the universe. Later, the dark matter was added to the baryonic component, the amount of which is determined indirectly. This dark matter is manifested through the attractive force of gravity: its existence explains the flat rotation curves of spiral galaxies, the compactness of rich galaxy clusters, to which the virial theorem is applicable, and gravitational lensing effect. The nature of this matter has not been established, but its existence explains the observations.

Many observational works have been devoted to answering the cardinal question. These works use cosmological tests that determine the ratio $\Omega_{0}=\rho / \rho_{\mathrm{c}}$ (or deceleration parameter $q=\Omega_{0} / 2$ ). The review by [17] describes four different tests that allow one to reconcile theory and observation by comparing the theoretical dependence of some quantity on cosmological redshift $z$ with its observable behavior for different values of $\Omega_{0}$. One of the tests suggests observations at different distances of a "standard candle," that is, an object (for example, a galaxy) of known intrinsic luminosity. For a long time, no such object was available, since there is a variety of galaxy and quasar luminosities and the evolution of luminosities is not fully understood.

The value of the Hubble constant $H_{0}$ is very significant, since it is used to determine the critical density. The initial estimate of this constant by Hubble himself, $H_{0}=558 \mathrm{~km} / \mathrm{s} / \mathrm{Mpc}$, was overestimated by almost ten times, because he mistook bright objects, identified later by radio astronomy methods as HII regions, for stars, whereas the former are much brighter than the latter. As a result, luminosities were overestimated, distances derived from them were underestimated, hence the value of $H_{0}$ was overestimated, as established by A. Sandage in 1958 [18]. As time went on, the Hubble constant was refined. This was the focus of a series of papers by [19]. The distance scale was based on the brightest blue and red giants in galaxies, with reference to Cepheid variables. The results were summarized in a review [20], although it was noted that different methods give values of $H_{0}$ that differ by several times due to systematic errors of the methods. A detailed history of construction of cosmological models and creation of the observational basis of cosmology is available in the book [21].

The cosmological term was sometimes taken into account without clarifying its meaning; more often it was ignored (see the review by [22]). The physical meaning to the cosmological term was given by E.B. Gliner [23]. Considering various forms of non-traditional energy-momentum tensors, he also interpreted the cosmological term as such a tensor corresponding to some substance, characterizing the substance as vacuum-like. Currently this substance is called "dark energy." Its repulsive effect leads to models of exponential time-dependent expansion of the universe. In the 1980s, these solutions were used in the theory of cosmological inflation, which describes the earliest stages of the evolution of the universe [24,25].

Substantial progress in determining cosmological parameters was achieved by technological advances in the end of 20th century, which provided improved instruments for ground-based telescopes, increased the sensitivity of receivers in different regions of the electromagnetic spectrum, and made possible launches of measuring devices operating in space. As a result, observational 
astrophysics, including cosmology, has become a multi-wavelength science. The decisive step towards the most adequate model of the real universe was made by two groups that used as a standard candle supernovae (SN) of type Ia, since the light curves of such SNs reliably determine their luminosity. Group one ([26]) using 10 SNs and group two ([27]) using 42 SNs have found that the main contribution to the critical density corresponds to the cosmological constant $\Lambda$. Both papers mentioned above describe the history of employing SN of this type as a standard candle. For example, this was done by [19], who used 16 SNs from the Coma Berenices and Virgo clusters to construct a Hubble diagram, with the calibration based on two close supernovae, 1937c in the galaxy IC4182 and 1954a in NGC4214. However, this was insufficient to make accurate conclusions.

Subsequently, the observations were extended to the highest redshifts, up to $\mathrm{z}=1$ and soon to $\mathrm{z}=1.8$ [28], which confirmed the conclusion of [26,27], and rejected other interpretations (see [22]). Confirmations were obtained by other types of observations as well. Therefore, the conclusion by $[26,27]$ that $\Lambda$ plays a significant role in the cosmos became a fundamental aspect of the Standard model that best describes the universe. Although refinement of the parameters of the model continues, results of the Wilkinson Microwave Anisotropy Probe (WMAP) [29] and Plank [30,31] missions have allowed us to specify the value of $H_{0}$ with an accuracy of $10 \%$ or even $7 \%$.

\section{Basic equations of homogeneous cosmological models}

In order to show the peculiarities of the Standard model we begin with a brief presentation of the general theory of homogeneous cosmological models.

\subsection{Space-time metric}

Any construction of a space-time model begins with the establishment of its metric. For cosmological models the metric in conventional notation has the common form: $\mathrm{d} s^{2}=c^{2} \mathrm{~d} t^{2}-\mathrm{d} l^{2}$. It is convenient to determine the following alternative functions, related by the equation $\operatorname{cs}_{k}^{2} \chi+k \operatorname{sn}_{k}^{2} \chi=1$ :

$$
\operatorname{sn}_{k} \chi=\left\{\begin{array}{ll}
\sin \chi & \text { for } k=1, \\
\chi & \text { for } k=0, \\
\operatorname{sh} \chi & \text { for } k=-1,
\end{array} \quad \operatorname{cs}_{k} \chi=\operatorname{sn}_{k}^{\prime} \chi= \begin{cases}\cos \chi & \text { for } k=1, \\
1 & \text { for } k=0 \\
\operatorname{ch} \chi & \text { for } k=-1\end{cases}\right.
$$

Then the cosmological principle, according to which everything in the universe on large scale at each moment is distributed uniformly and isotropically, is accepted, so that all points of space are equal. Everything that occurs in each of them and with respect to them is completely the same. This leads to the metric of space:

$$
\mathrm{d} l^{2}=R^{2}(\eta)\left[\mathrm{d} \chi^{2}+\operatorname{sn}_{k}^{2} \chi \mathrm{d} \omega^{2}\right], \quad \mathrm{d} \omega^{2}=\mathrm{d} \theta^{2}+\sin ^{2} \theta \mathrm{d} \varphi^{2},
$$

where $\eta$ and $\chi$ are dimensionless (conformal) time and spatial coordinates, with $k=1,0,-1$ corresponding to closed, flat, and open space, respectively, $R(\eta)$ is the radius of curvature, and $\mathrm{d} \omega^{2}$ is the metric on a sphere of unit radius, with angular coordinates $\theta$ and $\varphi$. The curvature, $k / R^{2}$, is constant at every moment over entire space. The only point of space that needs to be specified is the location of the observer (humanity), and the reference time, which is the current epoch.

Let us fix a moment $t$ and the corresponding conformal time $\eta$ and draw a ray with the beginning at the position of the observer $\mathrm{O}$, with direction determined by spherical angles $\theta=\theta_{0}, \varphi=\varphi_{0}$. There is a point on the sphere with a radius of unity which corresponds to this ray such that the distance $\mathrm{d} \omega=0$. Let us choose a point $P$ on the ray at distance $l$ from point $\mathrm{O}$ and with a spatial coordinate $\chi$, and draw a sphere, all points of which are at a distance $l$ from point $O$, so that it passes through the selected point $P$. Note that in the general case (if $k \neq 0$ ) the point $\mathrm{O}$ is not the center of the sphere (let us call it a quasi-center), the radius of the sphere, $r$, is not equal to $l$, and the ray is not a straight line. (In analogy, the radius of a parallel on the Earth's surface is not equal to the distance between the 
parallel and pole; the latter is not the center of the parallel, and a ray goes along the meridian, which is not a straight line.) The connection of $l$ and $r$ with a radius of curvature is expressed by the following equations:

$$
l=R(\eta) \chi, \quad r=R(\eta) \operatorname{sn}_{k} \chi
$$

wherein $l>r$ at $k=1, l<r$ at $k=-1$, and $l=r$ at $k=0$.

The adoption of a space metric defines all geometric properties of space. For example, the volume of space in a sphere whose points lay on the distance $l=R(\eta) \chi$ from $\mathrm{O}(\chi=0)$. A radius of the sphere is $R(\eta) \mathrm{sn}_{k}\left(\chi_{0}\right)$ for $k \neq 0$ is equal to

$$
V(\eta, \chi)=R^{3}(\eta) 4 \pi \int_{0}^{\chi_{0}} \operatorname{sn}_{k}^{2} \chi \mathrm{d} \chi=R^{3}(\eta) 2 \pi\left|\chi_{0}-\frac{1}{2} \operatorname{sn}_{k}\left(2 \chi_{0}\right)\right| .
$$

For $k=1$ the total volume of the space, $2 \pi^{2} R^{3}(\eta)$, is finite, since $\chi \leq \pi$. For $k=0$, it is formally necessary to replace $\operatorname{sn}_{k}\left(2 \chi_{0}\right)$ by its Taylor series representation up to the $\chi_{0}^{3}$ term.

The conformal time $\eta$ is connected with the usual time, which is fixed by the value of the density, by the equality $c \mathrm{~d} t=R(\eta) \mathrm{d} \eta$. In these variables, the metric takes the form of the Friedmann-Robertson-Walker metric:

$$
\mathrm{d} s^{2}=R^{2}(\eta)\left[\mathrm{d} \eta^{2}-\mathrm{d} \chi^{2}-\operatorname{sn}_{k}^{2} \chi \mathrm{d} \omega^{2}\right] .
$$

\subsection{The Friedmann Equations}

From the equations of the Einstein theory of gravitation (GR) with the metric (3), two equations for the radius of curvature can be derived, known as the Friedmann equations:

$$
\begin{gathered}
\ddot{R}=-\frac{4 \pi G}{3}\left(\rho+3 \frac{P}{c^{2}}\right) R+\frac{\Lambda c^{2}}{3} R, \\
\dot{R}^{2}=\frac{8 \pi G}{3} \rho R^{2}+\frac{\Lambda c^{2}}{3} R^{2}-k c^{2} .
\end{gathered}
$$

Here $\rho$ is the total mass density of matter and radiation, $P$ is their total pressure, and $\Lambda$ is the cosmological constant.

The terms with the cosmological constant in Eqs. (6)-(7) can be appended to the first term in each expression, which allows us to determine the total mass density and pressure, as well as the gravitational mass density:

$$
\rho_{\mathrm{t}}=\rho+\rho_{\Lambda}, \quad P_{\mathrm{t}}=P+P_{\Lambda}, \quad \rho_{\mathrm{g}}=\rho_{\mathrm{t}}+3 \frac{P_{\mathrm{t}}}{c^{2}} .
$$

To satisfy these relations, it is necessary to determine the density and pressure corresponding to the cosmological term as follows:

$$
\rho_{\Lambda}=\frac{\Lambda c^{2}}{8 \pi G}, \quad P_{\Lambda}=-\frac{\Lambda c^{4}}{8 \pi G} .
$$

It is negative pressure that produces repulsion.

Then the equations can be written in shorter form:

$$
\begin{gathered}
\ddot{R}=-\frac{4 \pi G}{3} \rho_{\mathrm{g}} R, \\
\dot{R}^{2}=\frac{8 \pi G}{3} \rho_{\mathrm{t}} R^{2}-k c^{2},
\end{gathered}
$$


or, for a scale factor $a=\frac{R}{R_{0}}=\frac{1}{1+z}$,

$$
\ddot{a}=-\frac{4 \pi G}{3} \rho_{\mathrm{g}} a, \dot{a}^{2}=\frac{8 \pi G}{3} \rho_{\mathrm{t}} a^{2}-\frac{k c^{2}}{R_{0}^{2}} .
$$

The past corresponds to the values $a<1$; at the current epoch $t=t_{0}, R=R_{0}, a=1$, and redshift $z=0$; and for the future $a>1,-1<z<0$. According to its definition, the scale factor is tied to the current epoch.

For compatibility of the equations, an additional condition is required:

$$
\dot{\rho}_{\mathrm{t}}=-3\left(\rho_{\mathrm{t}}+\frac{P_{\mathrm{t}}}{c^{2}}\right) H,
$$

where the new variable, $H=\frac{\dot{R}}{R}$, like the radius of curvature, depends only on time. We call it the "Hubble parameter." Its current value, $H_{0}$, is the Hubble constant. The relation (13) can be interpreted as the condition of adiabatic expansion of space along with its contents, since it implies that the differential of the total energy in volume $V$ satisfies

$$
\mathrm{d}\left(c^{2} \rho_{\mathrm{t}} V\right)=-P_{\mathrm{t}} \mathrm{d} V
$$

Equation (10) also yields an equation that the Hubble parameter obeys:

$$
\dot{H}=-H^{2}-\frac{4 \pi G}{3} \rho_{\mathrm{g}} .
$$

\subsection{Non-interacting components}

After the annihilation of electron-positron pairs, the composition of the universe became simpler, and since then its components have been the non-relativistic matter (including baryons and dark matter), radiation, neutrinos, and so-called dark energy (formerly called the vacuum), whose density and pressure are given by formulas (9). Of course, at high temperatures the matter was relativistic, but then its abundance was small. Similarly, due to the finiteness of the mass, at low temperatures neutrinos transformed from ultrarelativistic to relativistic (moderately or weakly) or even non-relativistic, but by then their mass fraction was small and difference of their masses from zero do not affect evolution of the universe [32]. Therefore, we assume that during the entire evolution of the universe over the period under consideration, the matter has not exerted pressure. This means that the matter has been non-relativistic (dust-like), while all kinds of neutrinos can be treated as ultra-relativistic.

We can assume that during this period the four components did not interact with each other. Dark energy in general does not interact with anything, while the interaction of cosmological neutrinos with matter essentially ceased before the annihilation epoch. Radiation interacted with matter, namely, free electrons and photons interacted until the end of the recombination epoch. However, after annihilation and establishment of equilibrium distributions, Compton (Thompson) scattering changes significantly neither the number of photons and electrons nor their energies. Therefore, the evolution of the components took place independently thereafter.

In view of the foregoing, the equations of state of the four indicated components: the dust matter $(d)$, the radiation $(r)$, neutrinos $(v)$, and dark energy $(\Lambda)$ are written in the form

$$
P_{\mathrm{d}}=0, \quad P_{\mathrm{r}}=\frac{c^{2}}{3} \rho_{\mathrm{r}}, \quad P_{v}=\frac{c^{2}}{3} \rho_{v}, \quad P_{\Lambda}=-c^{2} \rho_{\Lambda}
$$

The condition (13) is fulfilled for each non-interacting component separately:

$$
\dot{\rho}_{\mathrm{d}}=-3 \rho_{\mathrm{d}} H, \quad \dot{\rho}_{\mathrm{r}}=-4 \rho_{\mathrm{r}} H, \quad \dot{\rho}_{v}=-4 \rho_{v} H, \quad \dot{\rho}_{\Lambda}=0 .
$$


The equations are easily integrated, which provides the evolution of the densities of the components:

$$
\rho_{\mathrm{d}}=\frac{\rho_{\mathrm{d}}^{0} R_{0}^{3}}{R^{3}}=\frac{\rho_{\mathrm{d}}^{0}}{a^{3}}, \quad \rho_{\mathrm{r}}=\frac{\rho_{\mathrm{r}}^{0} R_{0}^{4}}{R^{4}}=\frac{\rho_{\mathrm{r}}^{0}}{a^{4}}, \quad \rho_{\nu}=\frac{\rho_{v}^{0} R_{0}^{4}}{R^{4}}=\frac{\rho_{v}^{0}}{a^{4}}, \quad \rho_{\Lambda}=\rho_{\Lambda}^{0} .
$$

192

Here, as above, the index 0 means belonging to the current epoch.

\subsection{Critical parameters}

In theory, the critical density, which plays an important role, and the fraction of all components in it are defined as:

$$
\rho_{\mathrm{c}}=\frac{3 H^{2}}{8 \pi G}, \quad \rho_{\mathrm{t}}-\rho_{\mathrm{c}}=k \frac{3 c^{2}}{8 \pi G R^{2}}, \quad \Omega_{\mathrm{t}}=\frac{\rho_{\mathrm{t}}}{\rho_{\mathrm{c}}}, \quad \Omega_{\mathrm{t}}-1=k \frac{c^{2}}{\dot{R}^{2}} .
$$

The sign of differences $\rho_{\mathrm{t}}-\rho_{\mathrm{c}}$ and $\Omega_{\mathrm{t}}-1$ coincides with the sign of $k$. If $\rho_{\mathrm{t}}-\rho_{\mathrm{c}}=0$ then $\Omega_{\mathrm{t}}-1=0$ and $k=0$. The shares of individual components are also determined:

$$
\Omega_{\mathrm{d}}=\frac{\rho_{\mathrm{d}}}{\rho_{\mathrm{c}}}, \quad \Omega_{\mathrm{r}}=\frac{\rho_{\mathrm{r}}}{\rho_{\mathrm{c}}}, \quad \Omega_{v}=\frac{\rho_{v}}{\rho_{\mathrm{c}}}, \quad \Omega_{\Lambda}=\frac{\rho_{\Lambda}}{\rho_{\mathrm{c}}}, \quad \Omega_{\mathrm{t}}=\Omega_{\mathrm{d}}+\Omega_{\mathrm{r}}+\Omega_{\Lambda} .
$$

The densities of the components are expressed in terms of the current critical density and their current shares in it:

$$
\rho_{\mathrm{d}}=\rho_{\mathrm{c}}^{0} \frac{\Omega_{\mathrm{d}}^{0}}{a^{3}}, \rho_{\mathrm{r}}=\rho_{\mathrm{c}}^{0} \frac{\Omega_{\mathrm{r}}^{0}}{a^{4}}, \rho_{v}=\rho_{\mathrm{c}}^{0} \frac{\Omega_{v}^{0}}{a^{4}}, \rho_{\Lambda}=\rho_{\mathrm{c}}^{0} \Omega_{\Lambda}^{0}, \quad \rho_{\mathrm{c}}^{0}=\frac{3 H_{0}^{2}}{8 \pi G} .
$$

Since radiation and neutrinos evolve in the same way, one can introduce their common density and pressure:

$$
\rho_{\mathrm{r} v}=\rho_{\mathrm{r}}+\rho_{v}=\rho_{\mathrm{c}}^{0} \frac{\Omega_{\mathrm{r} v}^{0}}{a^{4}}, \quad P_{\mathrm{r} v}=P_{\mathrm{r}}+P_{v}=\frac{c^{2}}{3} \rho_{\mathrm{r} v}, \quad \Omega_{\mathrm{r} v}=\frac{\rho_{\mathrm{r} v}}{\rho_{\mathrm{c}}} .
$$

Using introduced quantities, the second equation for the scale factor (12) is rewritten in the form:

$$
H=\frac{\dot{a}}{a}=\frac{H_{0}}{a^{2}} \sqrt{\Omega_{\mathrm{r} v}^{0}+\Omega_{\mathrm{d}}^{0} a+\Omega_{\Lambda}^{0} a^{4}-\frac{k c^{2}}{R_{0}^{2} H_{0}^{2}} a^{2}} .
$$

The first equation has already been taken into account in formulas (21). A solution of equation (23) represents an implicit dependency of the scaling factor on time. At the right hand of (23) under the square-root there is a fourth-order polynomial with respect to $a$. This form of the solution was obtained by Lemaitre [12]. Friedmann's solutions [10,11] did not take into account the radiation, so that the polynomial under the root was of the third order.

\subsection{Radiation, horizon, and distances}

The equation of motion of a photon along the ray $\theta=\theta_{0}, \varphi=\varphi_{0}$ toward us follows from the equality $\mathrm{d} s=0$, and connects its spatial and temporal coordinates: $\chi=\eta_{0}-\eta$. At the instant of emission $\chi_{\mathrm{e}}=\eta_{0}-\eta_{\mathrm{e}}$. Since $\eta_{\mathrm{e}} \geq 0$, it follows that $\chi_{\mathrm{e}} \leq \eta_{0}$. The equality $\chi_{\mathrm{e}}=\eta_{0}$ defines a spherical horizon; photons left this horizon at the initial moment. For $\chi_{\mathrm{e}}>\eta_{0}$ a photon, even released in our direction, still has not managed to reach us. This is a geometric horizon. There is also a physical horizon, which is the sphere of the last scattering during cosmological recombination. One can look beyond it: the theory of nucleosynthesis and the interpretation of distortions of the cosmic microwave background or relic radiation allow us to do so. However, it is impossible in principle to look behind the geometric horizon.

In cosmology, several concepts of distances can be introduced. McCrea [33] was the first to pay attention to this, and gave definitions of the distances. 
1. A metric distance $l$, which is the distance along the line of sight drawn from the observer with fixed angles (see formula (3)).

Other distances are determined by a common principle: the expression for any value in an expanding and, generally speaking, non-planar space, is written down, and then this expression is equated to the expression that would be true for the usual Euclidean space at a given distance. The distance is named according to the quantity for which formulas are written. Usually, the following distances from the observer are used (we define these at an arbitrary epoch, $t=t(\eta)$, but for the selected point where we, humanity, are located).

2. For the angular size. For the angular size. Let two signals issue at moment $t_{\text {source }}=t(\eta-\chi)$ from two points located from the observer at equal distance corresponding to the spatial coordinate $\chi$, and separated by an infinitesimally small angular distance, $\mathrm{d} \omega$. Let these signals arrive at the observer at moment $t=t(\eta)$. Then the linear distance between the points is $\mathrm{d} D_{\mathrm{ad}}=$ $R(\eta-\chi) \mathrm{sn}_{k}(\chi) \mathrm{d} \omega=l_{\mathrm{ad}} \mathrm{d} \omega$. This implies that the distance $l_{\mathrm{ad}}=R(\eta-\chi) \operatorname{sn}_{k} \chi$ is the radius of the sphere with quasi-center coinciding with the observer. The moment $\eta-\chi$ corresponds to points for which this distance is determined. The distance becomes zero for $\chi=0$ (at the point of the observer, as one might expect) and when $\chi=\eta$ (on the horizon). At some point the angular size has a minimum value. This means that the angular size of objects with the same linear size decreases in the beginning as the object recedes from the observer, and after passing the distance at which the angular size reaches its minimum value, it increases. This is due to the fact that in remote areas that correspond to earlier stages of expansion, the universe had a smaller scale, so that the lines of sight were closer to each other. Similarly, when a rail of a certain size is crossing from one pole of the Earth to another, first its angular size decreases, and then increases, since the meridians converge approaching the poles.

3. For the parallax $l_{\mathrm{pl}}=R(\eta) \mathrm{sn}_{k} \chi$, which is the radius of the sphere, but rather with a quasicenter at the point to which the distance is measured, and at the time of the measurement.

4. For the number of photons received by the observer from the source, taking into account the difference in passage of time at the source and the observer, $l_{\mathrm{nb}}=l_{\mathrm{pl}} \sqrt{R(\eta) / R(\eta-\chi)}$.

5. For the apparent bolometric luminosity (called also photometric distance) $l_{\mathrm{bb}}=l_{\mathrm{pl}} R(\eta) / R(\eta-\chi)$, where in addition to the difference in the passage of time, the loss of radiative energy due to redshift is taken into account. If the luminosity of an object located at the position corresponding to the radius of curvature $R(\eta)$ is equal to $L_{\mathrm{O}}$, then the observed luminosity according to the definition of distance derived from the bolometric brightness is equal to:

$$
L_{\mathrm{bb}}=\frac{L_{\mathrm{O}}}{4 \pi l_{\mathrm{bb}}^{2}} .
$$

To obtain the current values of these distances, it is necessary to substitute $\eta=\eta_{0}$, and $R\left(\eta_{0}\right)=R_{0}$. Modern distances are related as follows:

$$
l_{\mathrm{bb}}^{0}=l_{\mathrm{nb}}^{0} \sqrt{1+z}=l_{\mathrm{pl}}^{0}(1+z)=l_{\mathrm{ad}}^{0}(1+z)^{2}=R_{0} \operatorname{sn}_{k}(\chi)(1+z) .
$$

Since $z \geq 0$, in this chain of equalities the magnitude of the distances decreases from left to right.

The velocity of change of metric distance, which is the expansion rate at an arbitrary epoch, $\eta$, complies with the Hubble-Lemaître law:

$$
l=R(\eta) \chi, \quad v=\dot{l}=\dot{R} \chi=\frac{\dot{R}}{R} l=H l .
$$

The Hubble distance, at which the expansion velocity is equal to the speed of light, is $l_{\mathrm{H}}=c / H$; the current Hubble distance is $l_{\mathrm{H}}^{0}=c / H_{0}$. 
The relationship between speed and redshift is more complex than that between speed and distance [34]. At the current epoch the relation is:

$$
\frac{v}{c}=H_{0} \int_{0}^{z} \frac{\mathrm{d} z}{H} .
$$

This connection is model dependent and admits velocities greater than the speed of light, so that the cosmological redshift is not identical to the classical Doppler effect. The reason is that a photon changes its frequency not only at the instant of emission from a moving source, which is taken into account by the Doppler effect, but experiences a decrease in energy at each point of its flight to the observer due to the expansion of space, which occurs according to the appropriate model. The expansion occurs identically with respect to any point considered as a center. The existence of cosmological velocities higher than the speed of light does not contradict the theory of relativity, since the mutual receding of points occurs not because of their movement, but because of the expansion of space, across the complete span of which no signals are transmitted.

\section{The Standard model ( $\Lambda \mathrm{CDM})$}

\subsection{Model Parameters}

Modern cosmology has become a science based on observational data, which now have sufficient accuracy to construct a model that adequately describes the real universe. The most important aspect is the inference that space is very close to flat, which leads one to assume that $k=0$. In this case the radius of curvature is infinitely large and should not appear in expressions for quantities that have physical meaning. Therefore, as is often done, we adopt for its contemporary value the Hubble distance: $R_{0}=l_{\mathrm{H}}^{0}=c / H_{0}$. Then the metric (5) can be rewritten as:

$$
\mathrm{d} s^{2}=\left(l_{\mathrm{H}}^{0}\right)^{2} a^{2}(\eta)\left[\mathrm{d} \eta^{2}-\mathrm{d} \chi^{2}-\chi^{2} \mathrm{~d} \omega^{2}\right] .
$$

Of all the cosmological parameters, the current temperature of the radiation, which is very close to thermal (deviations from a blackbody spectrum are of order $10^{-5} \div 10^{-4}$ ) and called the cosmic microwave or relict background, has been determined with the greatest in cosmology accuracy: its value is $T_{0}=2.7277 \mathrm{~K}$. At an arbitrary epoch corresponding to redshift $z, T=T_{0} / a=T_{0}(1+z)$. The temperature of the neutrinos is connected to that of the radiation as $T_{v}=\sqrt[3]{4 / 11} T=0.71377 T$, $T_{v}^{0}=1.9469 \mathrm{~K}$. The coefficient is obtained from the consideration that, due to the adiabatic expansion, the entropy of the total mixture of matter and radiation does not change, while during the annihilation of electron-positron pairs their entropy passes to the radiation [35]. The entropy of the neutrino gas depends only on its temperature, and does not change.

Since radiation and neutrinos are ultrarelativistic, their mass densities are proportional to the fourth power of their temperature. For radiation according to the Stefan-Boltzmann formula,

$$
\rho_{\mathrm{r}}^{0}=\frac{a_{\mathrm{SB}}}{c^{2}} T_{0}^{4}=4.66 \cdot 10^{-34} \mathrm{~g} / \mathrm{cm}^{3},
$$

where $a_{\mathrm{SB}}=\left(8 \pi^{5} h / 15 c^{3}\right)\left(k_{\mathrm{B}} / h\right)^{4}$ is called the Stefan constant. For six types of neutrinos, which are fermions rather than bosons,

$$
\rho_{v}^{0}=6 \cdot \frac{7}{8} \cdot \frac{a_{\mathrm{SB}}}{c^{2}}\left(T_{v}^{0}\right)^{4}=6.35 \cdot 10^{-34} \mathrm{~g} / \mathrm{cm}^{3} .
$$

Together, radiation and neutrinos have a density

$$
\rho_{\mathrm{r} v}^{0}=1.10 \cdot 10^{-33} \mathrm{~g} / \mathrm{cm}^{3} .
$$


The Hubble constant, according to the latest definitions, is known to within several percent: $H=70 \pm 3$ $\mathrm{km} / \mathrm{s} / \mathrm{Mpc}[29,31]$. Here we adopt $H_{0}=70 \mathrm{~km} / \mathrm{s} / \mathrm{Mpc}=2.27 \cdot 10^{-18} 1 / \mathrm{s}$, so that the current critical density and the Hubble distance are equal to:

$$
\begin{gathered}
\rho_{\mathrm{c}}^{0}=\frac{3 H_{0}^{2}}{8 \pi G}=9.207 \cdot 10^{-30} \mathrm{~g} / \mathrm{cm}^{3} \\
l_{\mathrm{H}}^{0}=\frac{c}{H_{0}}=1.3215 \cdot 10^{28} \mathrm{~cm}=14.2 \mathrm{G} \text { light yrs }=4.2828 \mathrm{Gpc}
\end{gathered}
$$

Current relative fractions of radiation, neutrinos, and their sum are obtained as follows:

$$
\Omega_{\mathrm{r}}^{0}=5.06 \cdot 10^{-5}, \quad \Omega_{v}^{0}=6.90 \cdot 10^{-5}, \quad \Omega_{\mathrm{r} v}^{0}=1.196 \cdot 10^{-4}
$$

The main gravitational component of the mass of the universe, according to modern concepts, is dark energy; its share is estimated as $0.721 \pm 0.035$ [29]. Let's take the value $\Omega_{\Lambda}^{0}=0.72$, so that $\rho_{\Lambda}^{0}=6.63 \cdot 10^{-30} \mathrm{~g} / \mathrm{cm}^{3}$. Since space is flat, $\rho_{\mathrm{t}}=\rho_{\mathrm{c}}$ and $\Omega_{\mathrm{t}}=\Omega_{\mathrm{t}}^{0}=1$. The rest is a fraction of the dust, $\Omega_{\mathrm{d}}^{0}=1-\Omega_{\mathrm{r} v}^{0}-\Omega_{\Lambda}^{0}=0.27988 \approx 0.28$ and $\rho_{\mathrm{d}}^{0}=2.577 \cdot 10^{-30} \mathrm{~g} / \mathrm{cm}^{3}$.

Cosmological densities are very low, much lower than current densities in astronomical objects. Even in interstellar space, in each cubic centimeter there is an average of $\sim 1$ hydrogen atom. The densities of the cosmological components correspond to the following numbers of hydrogen atoms in a cubic meter (not $\mathrm{cm})$ :

$$
10^{6} \frac{\rho_{\mathrm{c}}^{0}}{m_{\mathrm{H}}}=5.5,10^{6} \frac{\rho_{\Lambda}^{0}}{m_{\mathrm{H}}}=4.0,10^{6} \frac{\rho_{\mathrm{d}}^{0}}{m_{\mathrm{H}}}=1.5,10^{6} \frac{\rho_{\mathrm{r}}^{0}}{m_{\mathrm{H}}}=2.8 \cdot 10^{-4}, 10^{6} \frac{\rho_{v}^{0}}{m_{\mathrm{H}}}=3.8 \cdot 10^{-4}, 10^{6} \frac{\rho_{\mathrm{r} v}^{0}}{m_{\mathrm{H}}}=6.6 \cdot 10^{-4},
$$

${ }^{68}$ where $m_{\mathrm{H}}=1.67 \cdot 10^{-24} \mathrm{~g}$. At the same time $1 \mathrm{~cm}^{3}$ contains $n_{\mathrm{ph}}^{0}=20.286 T_{0}^{3}=412$ relict photons and $6 \cdot \frac{3}{4} \cdot \frac{4}{11} n_{\mathrm{ph}}^{0}=674$ relict neutrinos. Using the density of dark energy, the current value of the cosmological constant is determined as $\Lambda=3 \frac{\Omega_{\Lambda}^{0}}{\left(l_{\mathrm{H}}^{0}\right)^{2}}=1.24 \cdot 10^{-56} \mathrm{~cm}^{-2}$. Note that during inflation this density was equal to the Planck density: $\rho_{\Lambda}=\frac{\Lambda c^{2}}{8 \pi G}=\rho_{\mathrm{Pl}}=\frac{c^{5}}{G^{2} \hbar}=5.1593 \cdot 10^{92} \mathrm{~g} / \mathrm{cm}^{3}$, so then it was $\Lambda_{\mathrm{Pl}}=9.6 \cdot 10^{66} \mathrm{~cm}^{-2}$.

\subsection{Basic dependencies}

Substituting into equation (23) $k=0$ and dividing the variables, we obtain the relationship between the time and scale factors. Using the relationship $c \mathrm{~d} t=l_{\mathrm{H}}^{0} a(\eta) \mathrm{d} \eta$, and the relationship between the time coordinate and $a$, we derive:

$$
\int_{0}^{a} \frac{a \mathrm{~d} a}{\sqrt{\Omega_{\mathrm{r} v}^{0}+\Omega_{\mathrm{d}}^{0} a+\Omega_{\Lambda}^{0} a^{4}}}=H_{0} t, \quad \int_{0}^{a} \frac{\mathrm{d} a}{\sqrt{\Omega_{\mathrm{r} v}^{0}+\Omega_{\mathrm{d}}^{0} a+\Omega_{\Lambda}^{0} a^{4}}}=\eta
$$

If we introduce the notation $\left(\Omega_{\mathrm{r} v}^{0}+\Omega_{\mathrm{d}}^{0}+\Omega_{\Lambda}^{0}=\Omega_{\mathrm{t}}=1\right)$,

$$
H_{\Lambda}=H_{0} \sqrt{\Omega_{\Lambda^{\prime}}^{0}} \quad x_{0}=\left(\frac{\Omega_{\Lambda}^{0}}{\Omega_{\mathrm{r} v}^{0}}\right)^{1 / 4}, \quad \beta=\frac{\Omega_{\mathrm{d}}^{0}}{\left(\Omega_{\mathrm{r} v}^{0}\right)^{3 / 4}\left(\Omega_{\Lambda}^{0}\right)^{1 / 4}}, \quad \eta_{*}=\left(\Omega_{\mathrm{r} v}^{0} \Omega_{\Lambda}^{0}\right)^{-1 / 4},
$$

and make the change of variable $a=x / x_{0}$, then the equation (23) will be transformed into

$$
H=\frac{\dot{x}}{x}=H_{\Lambda} \frac{\sqrt{1+\beta x+x^{4}}}{x^{2}},
$$


and the relations between the variables take the form

$$
H_{\Lambda} t=I_{1}(x, \beta), \quad \eta=\eta_{*} I_{0}(x, \beta), \quad I_{j}(x, \beta)=\int_{0}^{x} \frac{x^{j} \mathrm{~d} x}{\sqrt{1+\beta x+x^{4}}} .
$$

The parameter of the integrals with variable upper limit is $\beta=265.69$. The values of the constants $H_{\Lambda}=59.397 \mathrm{~km} / \mathrm{s} / \mathrm{Mpc}=1.9249 \cdot 10^{-18} \mathrm{~s}^{-1}, x_{0}=8.8088, \eta_{*}=10.381$. The age of the universe according to the Standard model with the adopted values of the parameters is $t_{0}=I_{1}\left(x_{0}, \beta\right) / H_{\Lambda}=$ $4.33 \cdot 10^{17} \mathrm{~s}=13.722 \mathrm{Gyr}$.

The two integrals are computed numerically, although approximate representations of the integrals are possible as well. For small $x$, relatively simple formulas can be obtained:

$$
\begin{gathered}
I_{0}(x, \beta) \sim 2 \frac{x}{q}-\frac{1}{35} \frac{x^{5}}{r} \frac{1}{q^{2}}\left(5+\frac{10}{q}+\frac{12}{q^{2}}+\frac{8}{q^{3}}\right)+\frac{x^{9}}{1716 r^{3} q^{2}}\left(99+\frac{198}{q}+\frac{252}{q^{2}}+\frac{216}{q}+\frac{80}{q^{4}}-\frac{96}{q^{5}}-\frac{192}{q^{6}}-\frac{128}{q^{7}}\right), \\
I_{1}(x, \beta) \sim \frac{2}{3} \frac{q+1}{q^{2}} x^{2}-\frac{x^{6}}{63 r q^{2}}\left(7+\frac{14}{q}+\frac{18}{q^{2}}+\frac{16}{q^{3}}+\frac{8}{q^{4}}\right)+ \\
+\frac{x^{10}}{2860 r^{3} q^{2}}\left(143+\frac{286}{q}+\frac{374}{q^{2}}+\frac{352}{q^{3}}+\frac{200}{q^{4}}-\frac{32}{q^{5}}-\frac{224}{q^{6}}-\frac{256}{q^{7}}-\frac{128}{q^{8}}\right) .
\end{gathered}
$$

Here $r=\sqrt{1+\beta x}, q=1+r$. These formulas represent the integral $I_{1}$ with a relative discrepancy of $10^{-6}$ for $x \leq 1.9,10^{-5}$ for $x \leq 2.5$, and $10^{-4}$ for $x \leq 3.2$. The accuracy of the formula for $I_{0}$ is somewhat higher: the value of $10^{-6}$ is already achieved for $x \leq 2.1,10^{-5}$ for $x \leq 2.9$, and $10^{-4}$ for $x \leq 3.6$.

For large values of the argument, the behavior of the integrals is substantially different. The integral $I_{0}$ from $x \rightarrow \infty$ has a finite limit, while $I_{1}$ tends to infinity. Approximately, they can be represented as follows:

$$
\begin{gathered}
I_{0}(x, \beta) \sim I_{0}(\infty, \beta)-\frac{1}{x}\left(1+\frac{\beta}{x^{3}}\right)^{1 / 2} F\left(1, \frac{5}{6}, \frac{4}{3},-\frac{\beta}{x^{3}}\right), I_{1}(x, \beta) \sim \ln x+S_{0}\left(\beta / x^{3}\right)+P\left(x_{*}, \beta\right), \\
P\left(x_{*}, \beta\right)=I_{1}\left(x_{*}, \beta\right)-\ln x_{*}-S_{0}\left(\beta / x_{*}^{3}\right), S_{0}(u)=\frac{1}{3} \sum_{n=1}^{\infty} \frac{(2 n-1) ! !}{n(2 n) ! !}(-u)^{n} .
\end{gathered}
$$

Here $F(a, b, c, x)$ is the hypergeometric function. For $x_{*}$, we can take the value of 10 . For $\beta=265.69$, the values of the integrals in the last formulas are: $I_{0}(\infty, \beta)=0.42880, I_{1}(10, \beta)=0.94380$, and $P(10, \beta)=-1.3992$. Calculations using formula (41) give the value of $I_{0}(x, \beta)$ with five significant digits when $x \geq 7.3$, and with (42) five significant digits of $I_{1}(x, \beta)$ are obtained when $x \geq 8.5$.

It should be emphasized that the scale factor $a$ and the redshift $z$ are tied to the current epoch, and that they change with increasing age of the universe. At the same time, the variable $x$ is associated only with time $t$ (through the radius of the curvature $R$ ), while the parameters $\beta, H_{\Lambda}$, and $\eta_{*}$ are strictly constant. Indeed, products $M_{\mathrm{d}}=\frac{4 \pi}{3} \rho_{\mathrm{d}} R^{3}=2.49 \cdot 10^{55} \mathrm{~g}$ (the mass of dust matter in a sphere of radius $R)$ and $W=4 \pi \rho_{\mathrm{rv}} R^{4}=4.22 \cdot 10^{80} \mathrm{~g} \cdot \mathrm{cm}$ do not depend on time, as is also the case for the density $\rho_{\Lambda}=\rho_{\Lambda}^{0}$, which is proportional to the cosmological constant $\Lambda$. These values can be used to express the variable $x$ and other parameters:

$$
x=\left(\frac{4 \pi \rho_{\Lambda}}{W}\right)^{1 / 4} R, H_{\Lambda}=\sqrt{\frac{\Lambda}{3}} c, \beta=\frac{3 M_{\mathrm{d}}}{W^{3 / 4}\left(4 \pi \rho_{\Lambda}\right)^{1 / 4}}, \eta_{*}=\left(\frac{9}{16 \pi} \frac{c^{4}}{G^{2} W \rho_{\Lambda}}\right)^{1 / 4}=\left(\frac{9}{2} \frac{c^{2}}{G W \Lambda}\right)^{1 / 4} .
$$

The variable $\eta$ is directly connected with time and expressed through $\eta_{*}$ and $x$. 
Table 1. Epochs of equality of densities and forces.

\begin{tabular}{|l|l|l|l|l|l|l|}
\hline Epoch & $x$ & $z$ & $\eta$ & $t / t_{0}$ & $t$ Gyrs & $t_{0}-t$ \\
\hline$\rho_{\mathrm{d}}=\rho_{\mathrm{r}}$ & 0.00159 & 5529 & 0.0151 & $1.34 \cdot 10^{-6}$ & $1.85 \cdot 10^{-5}$ & 13,7 \\
$\rho_{\mathrm{d}}=\rho_{v}$ & 0.00217 & 4057 & 0.0200 & $2.41 \cdot 10^{-6}$ & $3.31 \cdot 10^{-5}$ & 13.7 \\
$\rho_{\mathrm{d}}=2 \rho_{\mathrm{r}}$ & 0.00319 & 2764 & 0.280 & $4.90 \cdot 10^{-6}$ & $6.72 \cdot 10^{-5}$ & 13.7 \\
$\rho_{\mathrm{d}}=\rho_{\mathrm{r} v}$ & 0.00376 & 2339 & 0.0324 & $6.64 \cdot 10^{-6}$ & $9.11 \cdot 10^{-5}$ & 13.7 \\
$\rho_{\mathrm{d}}=2 \rho_{v}$ & 0.00434 & 2028 & 0.365 & $8.59 \cdot 10^{-6}$ & $1.72 \cdot 10^{-4}$ & 13.7 \\
$\rho_{\mathrm{d}}=2 \rho_{\mathrm{r} v}$ & 0.00752 & 1169 & 0.0572 & $2.27 \cdot 10^{-5}$ & $3.11 \cdot 10^{-4}$ & 13.7 \\
$\rho_{\mathrm{r} v}=\rho_{\Lambda}$ & 1.0000 & 7.809 & 1.198 & 0.0488 & 0.669 & 13.0 \\
$\rho_{\mathrm{d}}=2 \rho_{\Lambda}$ & 5.1025 & 0.7264 & 2.7138 & 0.5261 & 7.219 & 6.5 \\
$\rho_{\mathrm{g}}=0$ & 5.1050 & 0.7255 & 2.7144 & 0.5264 & 7.224 & 6.5 \\
$\rho_{\mathrm{d}}=\rho_{\Lambda}$ & 6.4288 & 0.3702 & 2.983 & 0.7043 & 9.66 & 4.06 \\
Current & 8.8088 & 0 & 3.32 & 1 & 13.7 & 0 \\
\hline
\end{tabular}

\subsection{Roles of components at different epochs}

In the expressions for total mass density

$$
\rho_{\mathrm{t}}=\rho_{\mathrm{c}}=\rho_{\mathrm{d}}+\rho_{\mathrm{r} v}+\rho_{\Lambda}=\rho_{\mathrm{c}}^{0} \Omega_{\Lambda}^{0} \frac{1+\beta x+x^{4}}{x^{4}}
$$

and gravitational mass density

$$
\rho_{\mathrm{g}}=\rho_{\mathrm{d}}+2 \rho_{\mathrm{r} v}-2 \rho_{\Lambda}=\rho_{\mathrm{c}}^{0} \Omega_{\Lambda}^{0} \frac{2+\beta x-2 x^{4}}{x^{4}}
$$

the mass density of dark energy is constant, while others decrease with time. Therefore, at different epochs the components have played different roles.

At certain points in time, the densities become equal. Since the components give different contributions to the gravitational mass density - the radiation gives a double positive contribution, and the vacuum gives a double negative one - their effect on the gravitation is different at different times. All of these moments are given in Table 1, which lists the values of the parameter $x$, the redshift $z$, and the coordinate $\eta$, the fraction of the full age and the age of the universe itself at the corresponding moments, as well as the time elapsed from these moments to the present epoch. The gravitational mass density becomes zero at a value of $x$ determined by the equation $x^{4}-(\beta / 2) x-1=0$. Moments when $\rho_{\mathrm{d}}=\rho_{\Lambda}$ and when $\rho_{\mathrm{g}}=0$ almost coincide, because the radiation and neutrino densities are small at these moments. The moment when $\rho_{\mathrm{r} v}=\rho_{\Lambda}$ corresponds to the time when $x$ is very close to 1 .

\subsection{Distances, speeds, acceleration: past, current, and future}

In the flat model, $\mathrm{sn}_{0}(\chi)=\chi$, the quasicenter and real center of spheres coincide, the parallax distance and the radius of a sphere are equal to the metric distance: $l_{\mathrm{pl}}=r=l$. In the Standard model the expressions for $l / l_{H}^{0}$ and the dimensionless velocity of the expansion $v / c$ coincide as well. Indeed, at any moment:

$$
\frac{v}{c}=\frac{i}{c}=\frac{H}{c} l=\frac{l}{l_{H}} \text {. }
$$

In the Standard model the metric distance from the observer in the current universe to a location with coordinate $\chi$ is given by the formula following from (26) and (38):

$$
l^{0}=R\left(\eta_{0}\right) \chi=R_{0} a\left(\eta_{0}\right) \chi=l_{\mathrm{H}}^{0}\left(\eta_{0}-\eta\right)=l_{\mathrm{H}}^{0} \eta_{*}\left[I_{0}\left(x_{0}, \beta\right)-I_{0}(x, \beta)\right] .
$$



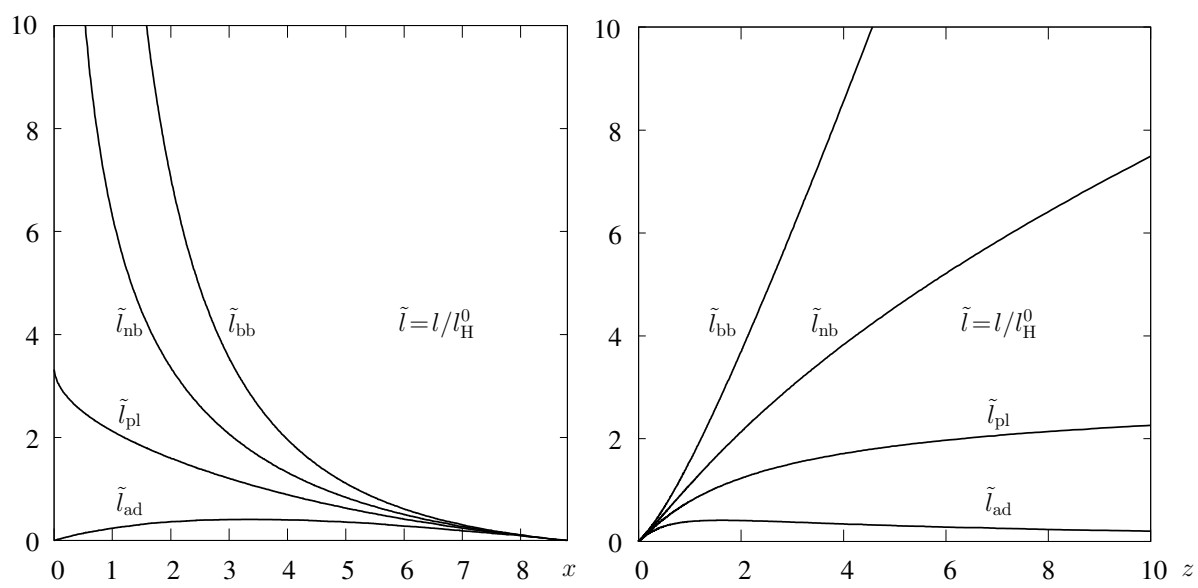

Figure 1. Distances as functions of $x$ (left) and $z$ (right).

Table 2. Epochs associated with the characteristic values of redshift.

\begin{tabular}{|l|l|l|l|l|l|}
\hline$x$ & $z$ & $\eta$ & $t / t_{0}$ & $t$ Gyrs & $t_{0}-t$ \\
\hline 0.0088000 & 1000 & 0.064629 & $2.9659 \cdot 10^{-5}$ & $4.0697 \cdot 10^{-4}$ & 13.721 \\
0.017582 & 500 & 0.10796 & $9.4743 \cdot 10^{-5}$ & $1.3000 \cdot 10^{-3}$ & 13.720 \\
0.087215 & 100 & 0.30606 & $1.2021 \cdot 10^{-3}$ & 0.016494 & 13.705 \\
0.17272 & 50 & 0.45696 & $3.4279 \cdot 10^{-3}$ & 0.047036 & 13.675 \\
0.80080 & 10 & 1.0642 & 0.034925 & 0.47924 & 13.242 \\
0.97875 & 8 & 1.1841 & 0.047233 & 0.64811 & 13.074 \\
3.3491 & 1.6302 & 2.2320 & 0.29360 & 4.0288 & 9.6930 \\
3.6350 & 1.4233 & 2.3224 & 0.33008 & 4.5293 & 9.1925 \\
2.2022 & 3 & 1.8084 & 0.15891 & 2.1806 & 0.11541 \\
\hline
\end{tabular}

The equalities (25) can be rewritten as

$$
l_{\mathrm{bb}}^{0}=l_{\mathrm{nb}}^{0} \sqrt{1+z}=l_{\mathrm{pl}}^{0}(1+z)=l_{\mathrm{ad}}^{0}(1+z)^{2}=R_{0} \chi(1+z)=l^{0}(1+z) .
$$

In what follows, we refer mainly to current values and use dimensionless distances, measuring them in terms of the Hubble distance according to the scheme $\tilde{l}=l / l_{\mathrm{H}}^{0}$. Therefore, all distances are expressed (as $v / c$ in (46)) via the metric distance:

$$
\tilde{l}_{\mathrm{pl}}=\frac{v}{c}=\tilde{l}=\eta_{*}\left[I_{0}\left(x_{0}, \beta\right)-I_{0}(x, \beta)\right], \quad \tilde{l}_{\mathrm{ad}}=\tilde{l} a, \quad \tilde{l}_{\mathrm{nb}}=\frac{\tilde{l}}{\sqrt{a}}, \quad \tilde{l}_{\mathrm{bb}}=\frac{\tilde{l}}{a}, \quad a=\frac{x}{x_{0}}=\frac{1}{1+z} .
$$

Figure 1 plots dependences of distances on the variable $x$ (left) and redshift $z$ (right).

Let us consider three additional moments in time corresponding to particular events. The first event was the phisical horizon (about $z=1000$ ), the second was when the angular size distance had its maximum value $(z=1.6302)$, and the third was when the current metric distance equaled the Hubble distance $(z=1.4233)$. These data, and also for comparison, the moments corresponding to several characteristic values of redshift, are given in Table 2, serving as a continuation of Table 1. Table 3 lists the values of the distances to the points indicated in Tables 1 and 2. The rate of change of the parallactic distance coincides with $v / c$, since this distance corresponds to the metric distance. The rates of recession of the remaining distances are determined from their definitions by differentiation with respect to time while keeping the spatial coordinate $\chi$ fixed. The expressions for these velocities are given in Table 4. 
Table 3. Distances to characteristic points of the Standard Model.

\begin{tabular}{|l|l|l|l|l|}
\hline$z$ & $\tilde{l}$ & $\tilde{l}_{\mathrm{ad}}$ & $\tilde{l}_{\mathrm{nb}}$ & $\tilde{l}_{\mathrm{bb}}$ \\
\hline$\infty$ & 3.322 & 0 & $\infty$ & $\infty$ \\
5529 & 3.307 & $5.981 \cdot 10^{-4}$ & 245.9 & 18288 \\
4057 & 3.302 & $8.138 \cdot 10^{-4}$ & 210.4 & 13401 \\
2764 & 3.294 & $1.192 \cdot 10^{-3}$ & 173.2 & 9108 \\
2339 & 3.290 & $1.406 \cdot 10^{-3}$ & 159.2 & 7700 \\
2028 & 3.286 & $1.619 \cdot 10^{-3}$ & 148.0 & 6667 \\
1169 & 3.265 & $2.790 \cdot 10^{-3}$ & 111.7 & 3821 \\
1000 & 3.258 & $3.255 \cdot 10^{-3}$ & 103.1 & 3261 \\
500 & 3.214 & $6.416 \cdot 10^{-3}$ & 71.95 & 1610 \\
100 & 3.016 & 0.02987 & 30.31 & 304.7 \\
50 & 2.865 & 0.05619 & 20.46 & 146.1 \\
10 & 2.258 & 0.2053 & 7.490 & 24.83 \\
8 & 2.138 & 0.2376 & 6.415 & 19.25 \\
7.809 & 2.125 & 0.2412 & 6.306 & 18.72 \\
3 & 1.514 & 0.3785 & 3.028 & 6.056 \\
1.630 & 1.090 & 0.4146 & 1.768 & 2.868 \\
1.423 & 1.000 & 0.4126 & 1.557 & 2.423 \\
0.7264 & 0.6086 & 0.3525 & 0.7996 & 1.051 \\
0.7255 & 0.6100 & 0.3524 & 0.7987 & 1.049 \\
0.3702 & 0.3397 & 0.2479 & 0.3977 & 0.4655 \\
0 & 0 & 0 & 0 & 0 \\
\hline
\end{tabular}

Table 4. Current velocities of recession at different distances.

\begin{tabular}{|c|c|c|c|c|}
\hline Metric & $\mathrm{ad}$ & $\mathrm{pl}$ & $\mathrm{nb}$ & $\mathrm{bb}$ \\
\hline$H_{0} l$ & $H\left(\eta_{0}-\chi\right) l_{\mathrm{ad}}$ & $H_{0} l_{\mathrm{pl}}$ & $\frac{3 H_{0}-H\left(\eta_{0}-\chi\right)}{2} l_{\mathrm{nb}}$ & {$\left[2 H_{0}-H\left(\eta_{0}-\chi\right)\right] l_{\mathrm{bb}}$} \\
\hline
\end{tabular}


The acceleration of the cosmological expansion is determined by the first equation in (12):

$$
\dot{v}=\ddot{l}=\frac{\mathrm{d}^{2}}{\mathrm{~d} t^{2}} l_{\mathrm{H}}^{0} a \chi=l_{\mathrm{H}}^{0} \ddot{a} \chi=\frac{\ddot{a}}{a} l=-\frac{4 \pi G}{3} \rho_{\mathrm{g}} l=H_{\Lambda}^{2} \frac{x^{4}-\beta x / 2-1}{x^{4}} l .
$$

As already mentioned, in the gravitational mass density $\rho_{\mathrm{g}}=\rho_{\mathrm{d}}+2 \rho_{\mathrm{r}}-2 \rho_{\Lambda}$ densities $\rho_{\mathrm{d}}$ and $\rho_{\mathrm{r}}$ decrease with increasing age of the universe, while $\rho_{\Lambda}=\rho_{\Lambda}^{0}$. Therefore, in the numerator of the last fraction in (50), the relative importance of the first term increases with time. At the present time $\left(x=x_{0}\right)$, the gravitational mass density is negative: $\rho_{\mathrm{g}}^{0}=\rho_{\mathrm{d}}^{0}+2 \rho_{\mathrm{r}}^{0}-2 \rho_{\Lambda}^{0}=-1.0677 \cdot 10^{-29} \mathrm{~g} / \mathrm{cm}^{3}$, so that the expansion occurs with an acceleration. But the acceleration at the current Hubble distance (speed equal to the speed of light) is only

$$
\dot{v}_{\mathrm{H}}^{0}=-\frac{4 \pi G}{3} \rho_{\mathrm{g}}^{0} l_{\mathrm{H}}^{0}=\frac{H_{0} c}{2}\left(2 \Omega_{\Lambda}^{0}-\Omega_{\mathrm{d}}^{0}-2 \Omega_{\mathrm{rv}}^{0}\right)=3.94 \cdot 10^{-8} \mathrm{~cm} / \mathrm{s}^{2} \approx 4 \AA
$$

In the distant future at $t \rightarrow \infty\left(\eta_{\infty}=4.4514\right)$

$$
a=\frac{1}{1+z} \sim\left(\frac{\Omega_{\mathrm{d}}^{0}}{4 \Omega_{\Lambda}^{0}}\right)^{1 / 3} e^{H_{\Lambda} t}=0.46000 e^{H_{\Lambda} t}, \quad x \sim 4.0520 e^{H_{\Lambda} t}, \quad \eta \sim \eta_{\infty}-2.5619 e^{-H_{\Lambda} t} .
$$

Thus, the scale of the universe will increase exponentially, so that a second inflation will take place, which we will discuss in more detail later. However, according to (52), an exponential expansion will really begin only at $t \sim t_{\Lambda}=1 / H_{\Lambda}$. The time scale is $1 / H_{0}=4.4081 \cdot 10^{17} \mathrm{~s}=13.969 \mathrm{Gyr}$, $t_{\Lambda}=1 / H_{\Lambda}=5.1950 \cdot 10^{17} \mathrm{~s}=16.462 \mathrm{Gyr}$. We also define the distance $l_{\Lambda}=c / H_{\Lambda}=l_{\mathrm{H}}^{0} / \sqrt{\Omega_{\Lambda}^{0}}=$ $1.5574 \cdot 10^{28} \mathrm{~cm}=5.0473 \mathrm{Gpc}$.

The speed of expansion of space at the Hubble distance is, by definition, equal to the speed of light. The velocity of recession of the Hubble distance is derived using equation (15):

$$
\dot{l}_{\mathrm{H}}=\frac{\mathrm{d}}{\mathrm{d} t} \frac{c}{H}=-\frac{c}{H^{2}} \dot{H}=\frac{c}{H^{2}}\left(H^{2}+\frac{4 \pi G}{3} \rho_{\mathrm{g}}\right)=c\left(1+\frac{1}{2} \frac{\rho_{\mathrm{g}}}{\rho_{\mathrm{c}}}\right)=\frac{c}{2} \frac{4+3 \beta x}{1+\beta x+x^{4}} .
$$

According to this formula, at the beginning of the expansion the velocity is close to the two speeds of light, decreasing with time, and in the distant future it will approach zero. Acceleration at the Hubble distance increases with time, but remains finite:

$$
\dot{v}_{\mathrm{H}}=-\frac{4 \pi G}{3} \rho_{\mathrm{g}} l_{\mathrm{H}}=-\frac{4 \pi G}{3} \rho_{\mathrm{g}} \frac{c}{H}=H_{\Lambda} c \frac{x^{4}-\beta x / 2-1}{x^{2} \sqrt{1+\beta x+x^{4}}} \rightarrow H_{\Lambda} c=5.77 \AA / \mathrm{s}^{2} .
$$

Acceleration of the distance itself is negative:

$$
\ddot{l}_{\mathrm{H}}=-\frac{c}{2} \frac{H_{\Lambda}}{x} \frac{\beta+16 x^{3}+9 \beta x^{4}}{\left(1+\beta x+x^{4}\right)^{3 / 2}} \sim-c H_{\Lambda} \frac{9}{2} \frac{\beta}{x^{3}} .
$$

From these formulas it is clear that the accelerations are of the same order as the product of the speed of light and the current Hubble constant, or the asymptotic value of the Hubble parameter: $c H_{0}=3 \cdot 10^{10} \cdot 2.27 \cdot 10^{-18}=6.81 \cdot 10^{-8} \mathrm{~cm} / \mathrm{s}^{2}, c H_{\Lambda}=5.77 \cdot 10^{-8} \mathrm{~cm} / \mathrm{s}^{2}$ (which coincides with the limit of (54)).

\subsection{Evolution of redshift and apparent luminosity}

As mentioned above, the scale factor $a$, and therefore the redshift $1+z=1 / a$ are tied to the epoch of observation. Therefore, the value of $z$ for each sufficiently distant object should change with increasing age of the universe. Therefore, luminosities of objects should change as well. A. Sandage drew attention to this problem. He calculated the changes for the model of "dust" with different values 
of $\Omega_{\mathrm{d}}^{0}$ ([36]). In the Appendix [37] to the paper [36], McVittie made the same calculations while adding a cosmological term. Later A.Loeb [38] (apparently independently) proposed to determine changes in the redshift $z$ of quasars using observations of the $L_{\alpha}$-forest with the 10-meter Keck telescope. He also transformed these changes into changes of the velocities of radiating objects. Such changes are known as the Sandage-Loeb effect.

Let us find the dependence of the change of $z$ on the age of the universe according to the Standard model. Since for this relation the dependence of the radius of curvature on time is significant, we will write $R(t)$ without changing the notation. The redshift of lines in the spectrum of some object at a location corresponding to time $t=t(\eta)$ from the beginning of the expansion, and observed at a position corresponding to the fixed time $t_{0}=t\left(\eta_{0}\right)$, is determined by the well known formula $1+z=R\left(t_{0}\right) / R(t)$. Then $z$ is uniquely related to time $t$, and equal to 0 at the observer's location, $z=0$. For the complete definition of $z$, both times should be specified as arguments, i.e., $z\left(t, t_{0}\right)$. However, this is traditionally not done, since the epoch of $t_{0}$ is fixed; in the past $z>0$ and in the future $-1<z<0$ with respect to $t_{0}$. At this point we adopt a more detailed designation.

After some time has passed, the age of the universe has increased and the epoch to which redshifts are attached has moved to the moment $t_{0}^{\prime}=t\left(\eta_{0}^{\prime}\right)$. Then an object at a given redshift has moved to time $t^{\prime}=t\left(\eta^{\prime}\right)$ without changing its spatial coordinate $\chi$. A connection between the moments of emission of radiation and its reception by the observer does not change in terms of the conformal coordinates, and the difference between the times of the observer and the object is preserved:

$$
\chi=\eta_{0}^{\prime}-\eta^{\prime}=\eta_{0}-\eta, \quad \eta_{0}^{\prime}-\eta_{0}=\eta^{\prime}-\eta .
$$

In particular, the infinitesimal displacements are equal as well: $\mathrm{d} \eta_{0}=\mathrm{d} \eta$. Using the relation $c \mathrm{~d} t=$ $R(t) \mathrm{d} \eta$ at times $t$ and $t_{0}$, we obtain the relation between the differentials of time and the derivative of one with respect to the other:

$$
\mathrm{d} t_{0}=\frac{R\left(t_{0}\right)}{c} \mathrm{~d} \eta_{0}=\frac{R\left(t_{0}\right)}{c} \mathrm{~d} \eta=\frac{R\left(t_{0}\right)}{c} \frac{c}{R(t)} \mathrm{d} t=\frac{R\left(t_{0}\right)}{R(t)} \mathrm{d} t, \quad \frac{\mathrm{d} t}{\mathrm{~d} t_{0}}=\frac{R(t)}{R\left(t_{0}\right)}=\frac{1}{1+z} .
$$

The last relation between the passage of time of the object and the observer has already been used in section 3.5 for the transformation from a parallactic distance to a distance measured according to the flux of photons.

To detect changes of $z$, one must measure shifts of lines in the spectrum of a source (with the same value of the $\chi$ coordinate) at different times. The difference between the times should be much smaller than the times themselves, so increments of values can be replaced by their differentials (infinitesimally small), and it is sufficient to determine the derivatives of the variables. Using (57) we find:

$$
\frac{\mathrm{d} R(t)}{\mathrm{d} t_{0}}=\frac{\mathrm{d} R(t)}{\mathrm{d} t} \frac{\mathrm{d} t}{\mathrm{~d} t_{0}}=\dot{R}(t) \frac{R(t)}{R\left(t_{0}\right)}, \quad \frac{\mathrm{d} R\left(t_{0}\right)}{\mathrm{d} t_{0}}=\dot{R}\left(t_{0}\right) .
$$

From the latter we obtain ([36])

$$
\frac{\mathrm{d} z}{\mathrm{~d} t_{0}}=\frac{\mathrm{d}(1+z)}{\mathrm{d} t_{0}}=\frac{\mathrm{d}}{\mathrm{d} t_{0}} \frac{R\left(t_{0}\right)}{R(t)}=\frac{\dot{R}\left(t_{0}\right)}{R(t)}-\frac{R\left(t_{0}\right)}{R^{2}(t)} \dot{R}(t) \frac{R(t)}{R\left(t_{0}\right)}=\frac{\dot{R}\left(t_{0}\right)}{R\left(t_{0}\right)} \frac{R\left(t_{0}\right)}{R(t)}-\frac{\dot{R}(t)}{R(t)}=H_{0}(1+z)-H .
$$

The dependence of $H$ on $z$ is derived if $x$ is substituted by $x_{0} /(1+z)$ in (37).

A change in the redshift will result in a change in the observed luminosity of objects. The rate of change of the photometric distance in the current epoch, as follows from the equalities (24), (48) (its boundary parts $l_{\mathrm{bb}}^{0}=l^{0}(1+z)$ ) and (59), is equal (in accordance with Table 4) to:

$$
\frac{\mathrm{d} l_{\mathrm{bb}}^{0}}{\mathrm{~d} t_{0}}=l^{0}(1+z)+l^{0} \frac{\mathrm{d} z}{\mathrm{~d} t_{0}}=H_{0} l^{0}(1+z)+l^{0}\left[H_{0}(1+z)-H\right]=\left(2 H_{0}-\frac{H}{1+z}\right) l_{\mathrm{bb}}^{0} .
$$


Then

$$
\dot{L}_{\mathrm{bb}}^{0}=-2 \frac{L_{\mathrm{O}}}{4 \pi\left(l_{\mathrm{bb}}^{0}\right)^{3}} \frac{\mathrm{d} l_{\mathrm{bb}}^{0}}{\mathrm{~d} t_{0}}=-2 \frac{L_{\mathrm{bb}}^{0}}{l_{\mathrm{bb}}^{0}} \frac{\mathrm{d} l_{\mathrm{bb}}^{0}}{\mathrm{~d} t_{0}}=-2 L_{\mathrm{bb}}^{0}\left(2 H_{0}-\frac{H}{1+z}\right), \frac{1}{H_{0}} \frac{\mathrm{d} \ln L_{\mathrm{bb}}^{0}}{\mathrm{~d} t_{0}}=-2\left(2-\frac{1}{1+z} \frac{H}{H_{0}}\right) .
$$

Figure 2 (for brevity, the derivative $\mathrm{d} z / \mathrm{d} t_{0}$ is denoted by $\dot{z}$ ) presents the dependencies of $\dot{z} / H_{0}$ and ratio $\dot{z} /\left[H_{0}(1+z)\right]$ on the (current) redshift $z$. First, the speed $\dot{z}$ is positive, i.e., $z$ increases; at $z=2.34$ the derivative $\dot{z}$ becomes zero. Between two zeros (at $z=0$ and $z=2.34$ ) at the point $z=1.06$ there is a maximum equal to 0.280 . The redshifts of more distant objects $(z>2.34)$ decrease; moreover, the rate of decrease grows rapidly with recession (increasing $z$ ): $\dot{z} / H_{0}$ is equal to $-0.98,-1.8,-8.4$, -30 at $z=4,5,10,20$ respectively. For the ratio $\dot{z} /\left[H_{0}(1+z)\right]$, the growth is less pronounced. The derivatives $\dot{z}$ and $\dot{z} /(1+z)$ are equal to zero at the same points, and $\dot{z} /(1+z)$ reaches the maximum at $z=0.726$ that is smaller than the maximum of $\dot{z}$.

Figure 2 also presents a graph of the dimensionless derivative of the logarithm of the apparent luminosity as a function of $z$. When $z=0$ this derivative equals -2 , which reflects a decrease in the solid angle of the source at the very beginning of the source's recession from the observer. At small $z$ the rate of decrease grows slightly; at $z=0.726$ it has maximum negative value, then decreases and becomes equal to zero at $z=13.2$. The apparent brightness of distant objects should increase with redshift at $z>13.2$. The effect is stronger for more distant objects, although it is unclear whether any radiating objects existed, since such redshifts correspond to times $<326$ million years from the beginning of the expansion, less than a fraction 0.0238 of the current age of the universe.

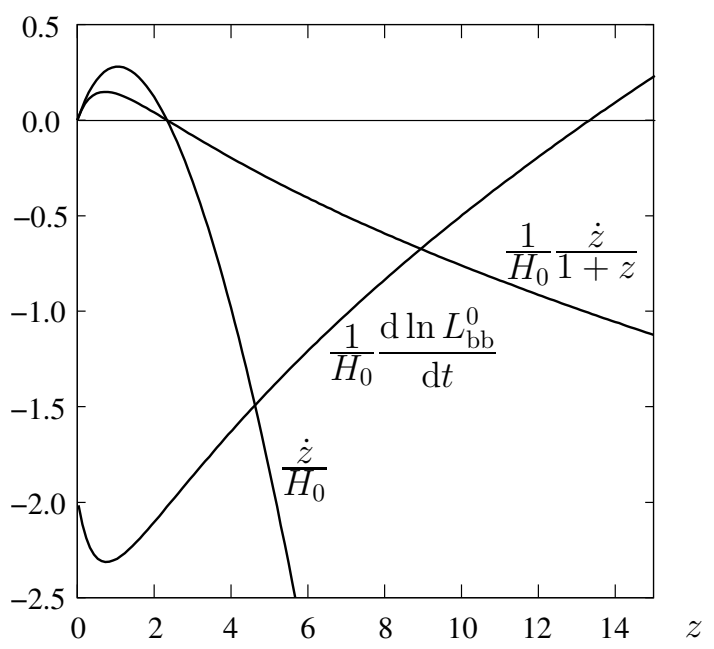

Figure 2. Changes in redshift and luminosity as a function of $z$.

It is most convenient to measure changes in all of these quantities when observing the $L_{\alpha}$-forest, which corresponds to shifts of the $L_{\alpha}$ line in the spectra of distant quasars due to gas clouds located along the path to them. These clouds can have peculiar radial velocities relative to the Hubble flow that can affect observed shifts of the line. However, values of these radial components most likely do not change significantly during the time between observations if they are separated within some decades up to hundred of years. The luminosities of objects do not change as well at least in average.

Despite the importance of this effect to test the theory, any possibility of observing it with modern instruments would require a very long time interval between observations, from hundreds to thousands years, since $\lambda\left(t_{0}\right) / \lambda(t)=1+z\left(t, t_{0}\right)$, then $\mathrm{d} \lambda_{0} / \lambda_{0}=\mathrm{d} z /(1+z)$, and

$$
\mathrm{d} t_{0}=\frac{1}{H_{0}} \frac{\mathrm{d} z /(1+z)}{\dot{z} /\left[H_{0}(1+z)\right]}
$$

For example, if we assume that the accuracy of measurements of a relative shift of lines is $\mathrm{d} \lambda / \lambda=$ $\mathrm{d} z /(1+z)=10^{-6}$ then for $z=4$, as seen from Fig. $2, \dot{z} /\left[H_{0}(1+z)\right] \approx-0.2$ and $\mathrm{d} t_{0} \sim 14 \cdot 10^{9}$. 
$10^{-6} / 0.2=3 \cdot 10^{3} \mathrm{yrs}-\mathrm{a}$ time interval which is insignificant on cosmological scales but longer than a human lifetime. For large $z$, the accuracy of measuring the position of the lines is less, so that we would need major technological progress to pursue such a method.

[39] estimates the possibility to detect the shift of lines due to cosmological expansion in the spectra of various objects at different wavelengths for different cosmological models when telescopes with ultra-large mirrors $(40-60 \mathrm{~m})$ become available, as is planned for the 2020s. It is alleged that a $42-\mathrm{m}$ telescope will be able to measure the shift with 4000 hour exposures separated by $40 \mathrm{yrs}$. The article also provides an overview of previous work on the question of changing redshift.

\section{The second inflation and the second horizon}

\subsection{Visible and invisible parts of the universe}

According to the theory of cosmological inflation, near the very beginning of the evolution of the universe, space expanded exponentially. The standard theory, as follows from (52), predicts that a positive cosmological constant causes an acceleration of space starting from a certain moment that leads to an exponential expansion, although at a much lower rate than during the first inflation. This new expansion generates a new concept: a second horizon.

The equation of motion of a photon traveling to the observer (that is, to us) is $\chi=\eta_{0}-\eta$; therefore, the place and time of its exit are related by the equality $\chi_{\mathrm{e}}=\eta_{0}-\eta_{\mathrm{e}}<\eta_{0}$. So, the equation of motion can be rewritten as follows: $\chi=\chi_{\mathrm{e}}+\eta_{\mathrm{e}}-\eta$. Therefore, the distance from the observer to the approaching photon is

$$
l_{\mathrm{rs}}=l_{\mathrm{H}}^{0} a(\eta)\left(\chi_{\mathrm{e}}+\eta_{\mathrm{e}}-\eta\right) .
$$

The parameter $\eta$ is limited. For $t=\infty$, it is equal to $\eta_{\infty}=4.4514$. The distance can only equal zero, $l_{\mathrm{rs}}=0$, if $\chi_{\mathrm{e}}+\eta_{\mathrm{e}}<\eta_{\infty}$. Then there is another limitation on the ability to observe objects in the universe: along with the first horizon there is a second one. The concept of two horizons was introduced by V. Rindler [40] and discussed in a number of papers, for example, in [41]. Here their kinematic characteristics are derived within the Standard model.

The first horizon is called geometric (we recall that the physical horizon is the sphere of last scattering at $z \approx 1000$ ), while the second horizon can be called the kinematic or dynamic horizon. Other names are also used, borrowed from the terminology of the theory of black holes. The geometric horizon is called the particle horizon, and the kinematic horizon is called the event horizon. These names were introduced by Rindler.

At an arbitrary epoch, $\eta$, the first and second horizons are determined by the equations

$$
\chi_{\mathrm{GHor}}=\eta=H_{0} \int_{0}^{a} \frac{\mathrm{d} a}{a^{2} H}=\eta_{*} I_{0}(x, \beta), \quad \chi_{\mathrm{KHor}}=\eta_{\infty}-\eta=H_{0} \int_{a}^{\infty} \frac{\mathrm{d} a}{a^{2} H}=\eta_{*}\left[I_{0}(\infty, \beta)-I_{0}(x, \beta)\right] .
$$

In Figure 3 positions of the geometric horizon are indicated on the ordinate axis. The lines corresponding to this horizon are parallel to the abscissa. They rise with time, reflecting expansion of the horizon. The second, kinematic horizon is shown by a straight line connecting the abscissa and the ordinate, which are equal to $\eta_{\infty}$, while its specific position corresponds to the time on the abscissa axis. The paths of photons coming toward us are represented by straight lines parallel to this straight line. Photons can start their journey from any point on the trajectory. Photons, for which $\eta_{\mathrm{e}}+\chi_{\mathrm{e}}<\eta_{\infty}$, that is, moving along straight lines lying below the straight line specified above, sooner or later will reach a place where the observer is located. For example, Figure 3 shows the paths of photons that have reached our position at time $\eta_{*}<\eta_{0}$ and at the current epoch $\eta_{0}$. If $\eta_{\mathrm{e}}+\chi_{\mathrm{e}}>\eta_{\infty}$, then photons with such coordinates never reach our location. According to the equality $\eta_{\mathrm{e}}+\chi_{\mathrm{e}}=\eta_{\infty}$, it would seem that the photon still must reach the observer at least over an infinite time, but even that is impossible. 


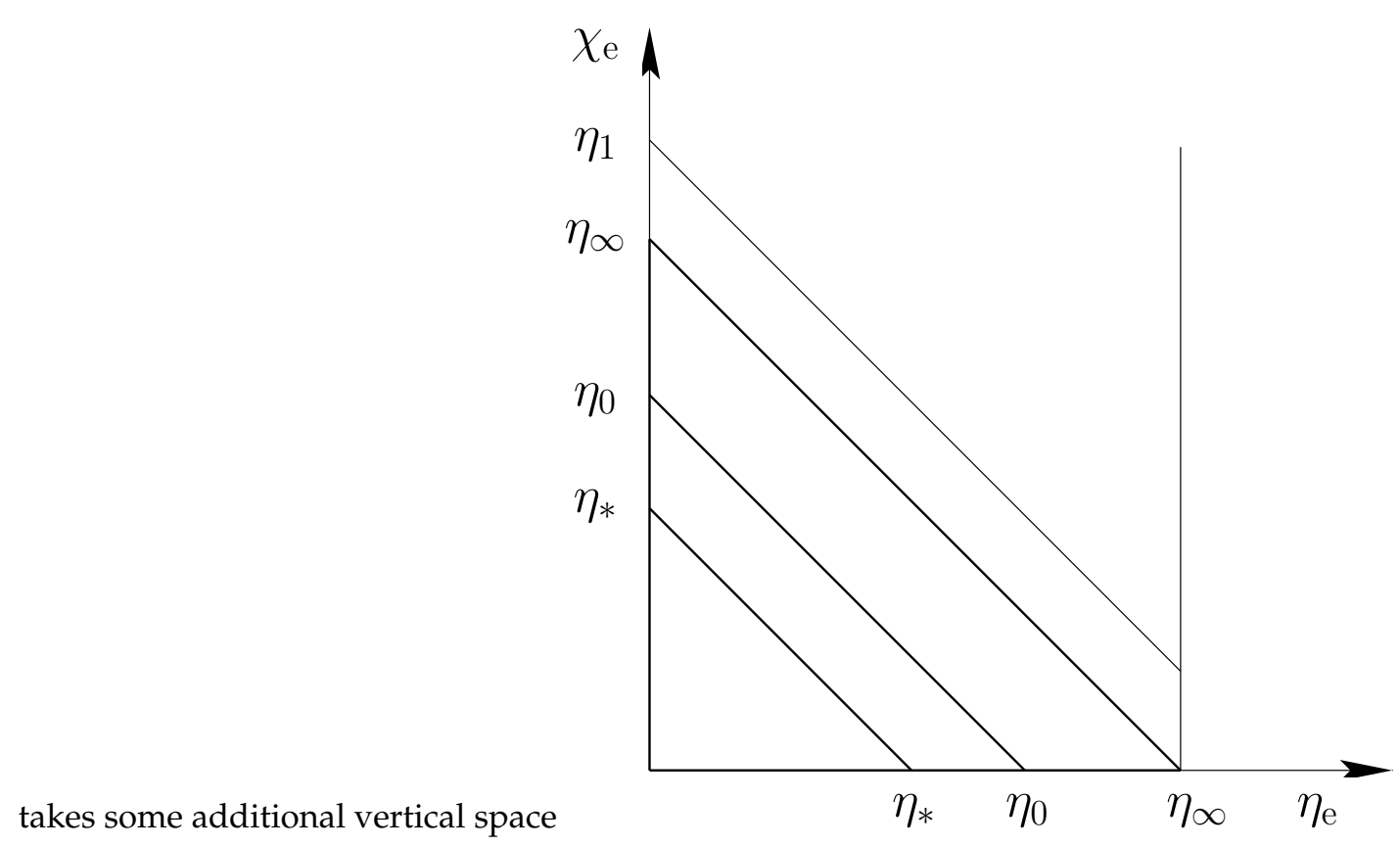

Figure 3. Visible and invisible parts of the universe.

From behind the first horizon, the radiation has not yet reached the observer. The second horizon separates the region of times and locations from which radiation cannot reach the observer, since the photons coming from there are moving away from the observer. This occurs because space expands at speeds higher than the speed of light, and these speeds increase with time. At the current time, we can see objects in the universe up to redshifts $z \approx 10$, but this corresponds to the past. We will never see objects located now at redshifts $z \geq 1.725$.

Indeed, if a photon is now emitted toward us from a place with coordinate $\chi_{0}$, then the distance to it at moment $\eta$ will be $l_{\mathrm{ph}}=l_{\mathrm{H}}^{0} a(\eta)\left(\chi_{\mathrm{o}}+\eta_{0}-\eta\right)$. This distance can become equal to zero at $\eta=\chi_{\mathrm{o}}+\eta_{0}$, and it must be the case that $\chi_{\mathrm{o}}+\eta_{0}<\eta_{\infty}$. Thus, the boundary of the coordinate $\chi_{\mathrm{o}}$ for photons emitted now is $\chi_{\lim }^{0}=\eta_{\infty}-\eta_{0}=1.13$. The values of $x_{\lim }^{0}=3.23, z_{\lim }^{0}=1.725$, and $l_{\lim }^{0}=4.84 \mathrm{Gpc}=l_{\mathrm{KHor}}^{0}$ correspond to this coordinate. The sphere of such a radius is the current second horizon. Thus, the radiation from the points now located at distances of $4.84 \mathrm{Gpc}$ from us will never reach us, even in the infinitely remote future. Figure 4 shows distances $l_{\mathrm{rs}}$ to the photons arriving at the observer at the epoch when $\eta=2$ (Figure 4 left), and at the current epoch (Figure 4 right). In Figure 5 these distances are given as a function of values of $x$ for cases where the sum of the coordinates of time and location of the photon emission is equal to $\eta_{\infty}$ (Figure 5 left) and larger than that (Figure 5 right). These figures also show curves reflecting the relationship between the time $\eta_{\mathrm{e}}$ and the location $\chi_{\mathrm{e}}$ of the photon emission.

Generally speaking, if a photon is emitted at a point where the expansion speed is greater than the speed of light, this does not necessarily mean that it will not reach us. Cosmological expansion occurs in the same way with respect to all points of space, and it starts after a period of inflation with a very high speed (formally infinite, according to formula (37), which defines the Big Bang), although in the beginning the expansion was slowing down. A photon emitted from far away, where the speed of expansion is large but closer than the horizon, still comes to us, because it gradually moves into layers of space expanding at a slower and slower rate. At some point its velocity toward us becomes zero, and then becomes negative, that is, it begins to approach us. However, it takes a long time for the photon to reach us. Consider, for example, a galaxy observed by us now at redshift $z=3$ : according to Table 2, it moves away from us with a speed of $H_{0} l=c \tilde{l}=1.51 c$, and earlier its speed was greater. At the same time, its radiation traveled to us for 11.5 billion years, that is, we see this galaxy as it was in 


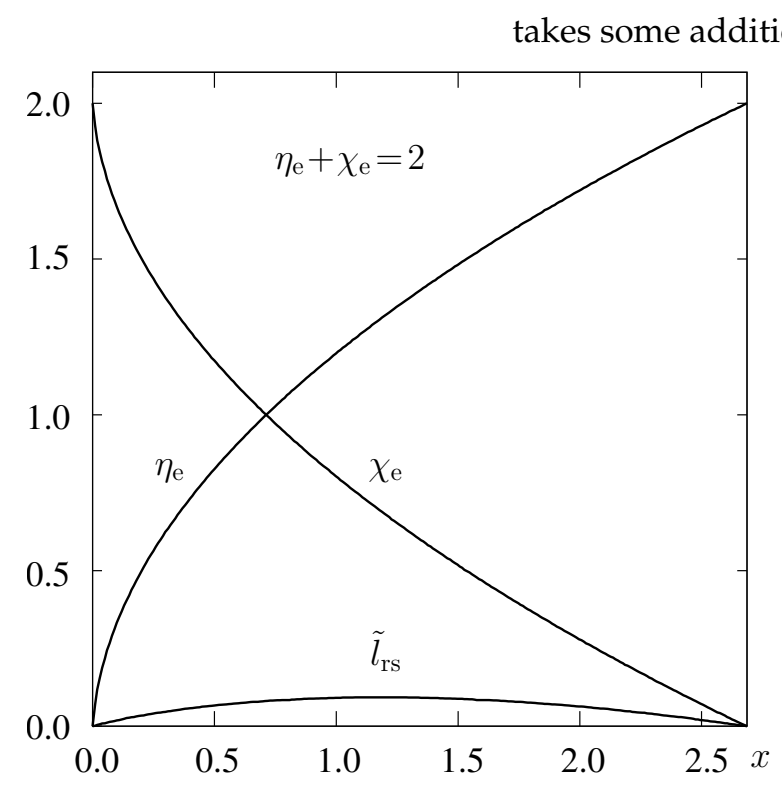

/

Figure 4. The paths of photons with arrival time at epochs: $\eta=2$ (left) and $\eta_{0}=3.3224$ (right).

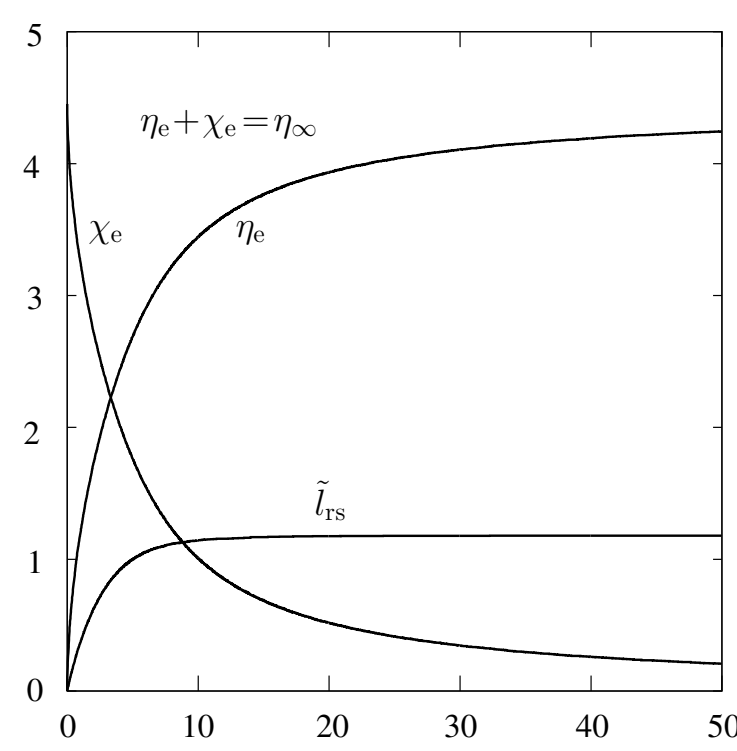

space

Figure 5. The paths of photons with "arrival" time at epochs: $\eta_{\infty}=4.4514$ (left) and $\eta=5$ (right).
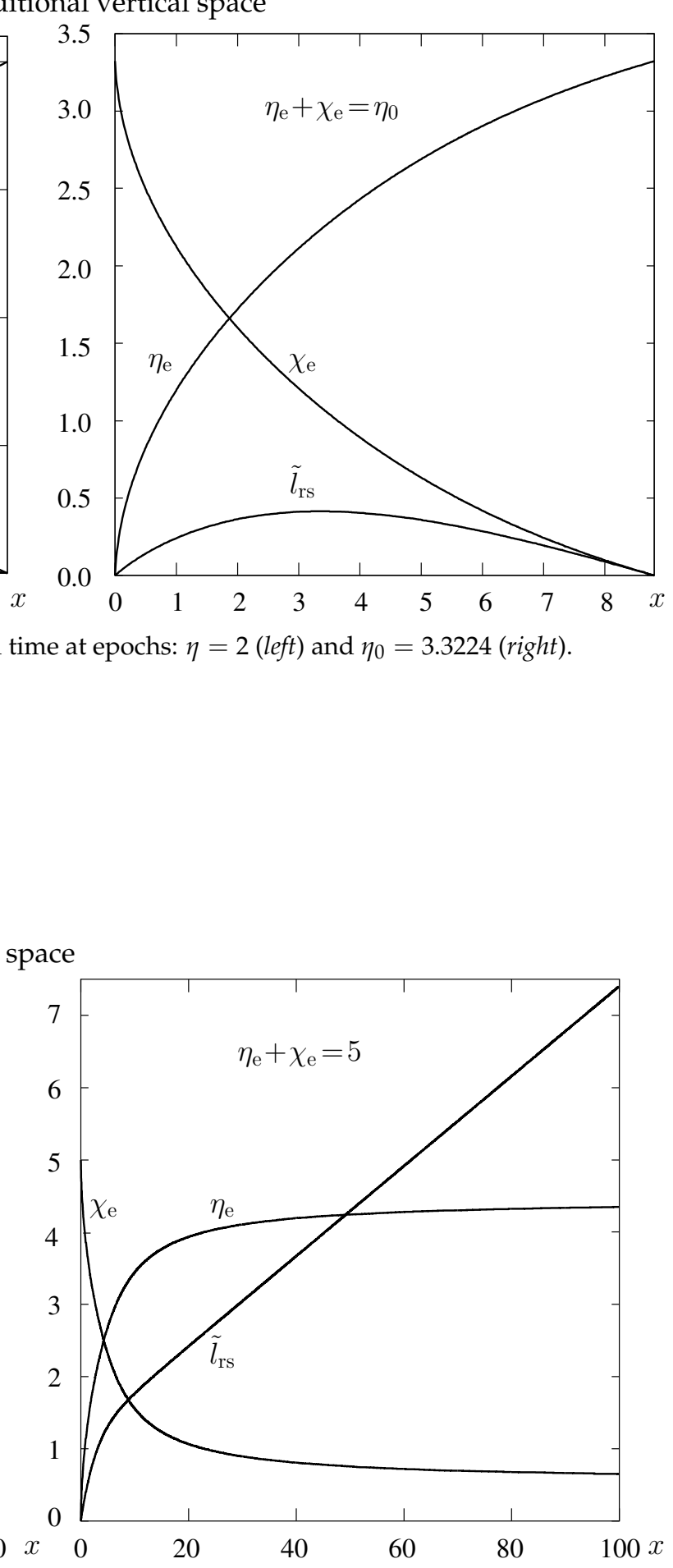
the distant past, when neither the Earth, nor even the Sun, existed (but the galaxies and stars of the previous generations had already formed).

Figure 4 shows that the distance of a photon emitted sufficiently early at first increases, which means expansion with a speed greater than the speed of light, faster than the photon speed. From the point where the distance reaches a maximum, the photon begins to approach and finally arrives at our location. However, as shown in Figure 5, this is not possible if $\eta_{\mathrm{e}}+\chi_{\mathrm{e}} \geq \eta_{\infty}$, even if the equality is true. Figure 5 left shows that a photon emitted at the second horizon, and which then travels along it, would not arrive at the observer after an infinite time; in fact, the photon only recedes along with the horizon. After an infinite time, such a photon will be at a distance $l_{\Lambda} \approx 5.0 \mathrm{Gpc}$, since the factor $\eta_{\infty}-\eta$ in the formula (63) at $\chi_{\mathrm{e}}+\eta_{\mathrm{e}}=\eta_{\infty}$ tends to zero if $t \rightarrow \infty$, while the factor $a(\eta) \rightarrow \infty$, but their product remains finite. A photon emitted at $\eta_{\mathrm{e}}+\chi_{\mathrm{e}}<\eta_{\infty}$ may, after a very long time, reach the current location of our civilization, but one emitted at $\eta_{\mathrm{e}}+\chi_{\mathrm{e}}>\eta_{\infty}$ will only recede from us, eventually exponentially fast. The reason for this is the accelerated expansion of space. Thus, galaxies located on the second horizon and behind it will forever disappear from our field of view. These statements follow from the formulas given below.

\subsection{Distances, velocities, and accelerations of horizons}

Distances to horizons at an arbitrary epoch $\eta$ according to equations (64) are defined by the formulas:

$$
\begin{gathered}
l_{\mathrm{GHor}}=l_{\mathrm{H}}^{0} a(\eta) \eta=l_{\Lambda} x I_{0}(x, \beta), \\
l_{\mathrm{KHor}}=l_{\mathrm{H}}^{0} a(\eta)\left(\eta_{\infty}-\eta\right)=l_{\Lambda} x\left[I_{0}(\infty, \beta)-I_{0}(x, \beta)\right] .
\end{gathered}
$$

The sum of the horizon conformal space coordinates is constant at all times, and the sum of the distances to them is proportional to the scale factor. Both horizons expand. The speed of the geometric horizon exceeds by the speed of light the velocity of the position where the horizon is located at time $i_{\mathrm{GHor}}=l_{\mathrm{H}}^{0} \dot{a} \eta+l_{\mathrm{H}}^{0} a \dot{\eta}=H l_{\mathrm{GHor}}+c$. It expands at an accelerating rate. In contrast, the velocity of the kinematic horizon is less than the speed of its location by the speed of light: $\dot{l}_{\mathrm{KHor}}=l_{\mathrm{H}}^{0} \dot{a}\left(\eta_{\infty}-\eta\right)-$ $l_{\mathrm{H}}^{0} a \dot{\eta}=H l_{\mathrm{KHor}}-c$, and its expansion slows down.

Asymptotes of distances to horizons and their velocities at $t \rightarrow \infty, a \rightarrow \infty, z \rightarrow-1$ are determined by taking into consideration that $I_{0}(\infty, \beta)=0.42880$ and $I_{0}(\infty, \beta)-I_{0}(x, \beta) \sim \frac{1}{x}\left(1-\frac{1}{8} \frac{\beta}{x^{3}}\right)$ :

$$
\begin{gathered}
l_{\mathrm{GHor}} \sim l_{\mathrm{H}}^{0} \eta_{*} I_{0}(\infty, \beta) a=5.9 \cdot 10^{28} a \mathrm{~cm} \sim 2.7 \cdot 10^{28} e^{H_{\Lambda} t} \mathrm{~cm} \rightarrow \infty, \\
l_{\mathrm{KHor}} \rightarrow \frac{c}{H_{\Lambda}}=1.56 \cdot 10^{28} \mathrm{~cm}=5.05 \mathrm{Gpc} .
\end{gathered}
$$

The current distance to the geometric horizon is $l_{\mathrm{GHor}}^{0}=l_{\mathrm{H}}^{0} \eta_{0}=3.32 l_{\mathrm{H}}^{0}=4.39 \cdot 10^{28} \mathrm{~cm}=14.2 \mathrm{Gpc}$. The velocity near the horizon is $v_{\mathrm{GHor}}^{0}=c \eta_{0}=3.32 c$, and the velocity of the expansion of the horizon is $\dot{l}_{\mathrm{GHor}}^{0}=4.32 \mathrm{c}$. The horizon will cross 4.32 light years in one year, which is equal to $1.33 \mathrm{pc}$, so that $1 \mathrm{Gpc}$ will be added to the current $14.2 \mathrm{Gpc}$ in $0.755 \cdot 10^{9}$ years if the speed of the horizon is equal to its current velocity, and in $0.741 \cdot 10^{9}$ years if the increase of the velocity is taken into account.

The current distance to the second horizon is $l_{\mathrm{KHor}}^{0}=l_{\mathrm{H}}^{0}\left(\eta_{\infty}-\eta_{0}\right)=1.49 \cdot 10^{28} \mathrm{~cm}=4.84 \mathrm{Gpc}$. The limit to this distance coincides with the Hubble limit: $l_{\mathrm{KHor}} \rightarrow \frac{l_{\mathrm{H}}^{0}}{\sqrt{\Omega_{\Lambda}^{0}}}=\frac{c}{H_{\Lambda}}=5.05 \mathrm{Gpc}$. The current speed of expansion of the location of this horizon is $c\left(\eta_{\infty}-\eta_{0}\right)=1.13 c$, and the speed of recession of the kinematic horizon from us is now $0.13 c$. 

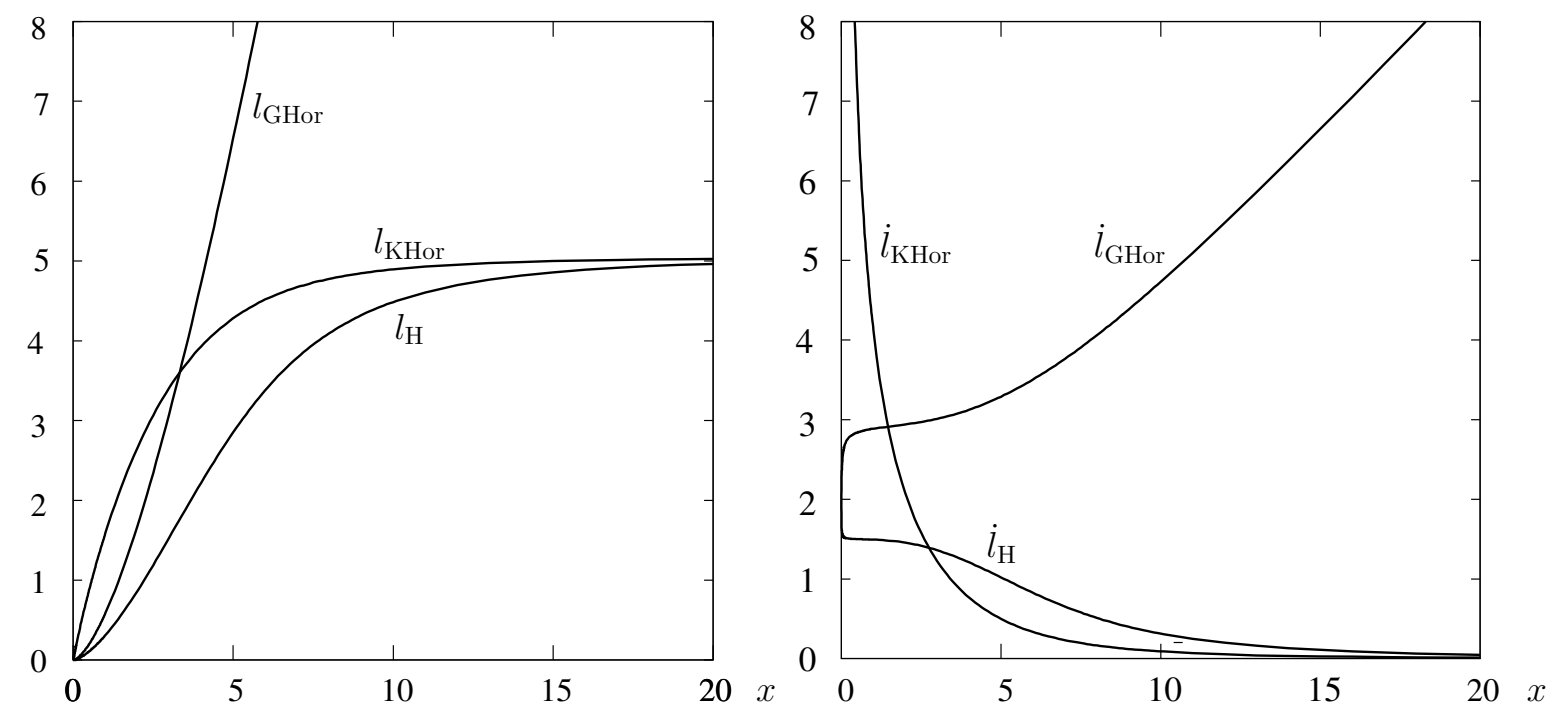

Figure 6. Left: Hubble distance and distances to the horizons in Gpc. Right: Speeds of change of distances in units of the speed of light. (right).

The velocities of the horizons at an arbitrary moment and their asymptotics for $t \rightarrow \infty$ and $x \sim 5.0 \cdot e^{H_{\Lambda} t} \rightarrow \infty$ are

$$
\begin{gathered}
\dot{l}_{\mathrm{GHor}}=l_{\mathrm{H}}^{0}(\dot{a} \eta+a \dot{\eta})=H l_{\mathrm{GHor}}+c=c\left[\frac{\sqrt{1+\beta x+x^{4}}}{x} I_{0}(x, \beta)+1\right] \sim c x I_{0}(\infty, \beta), \\
\dot{l}_{\mathrm{KHor}}=c\left[\frac{\sqrt{1+\beta x+x^{4}}}{x}\left[I_{0}(\infty, \beta)-I_{0}(x, \beta)\right]-1\right] \sim \frac{3}{8} \frac{\beta}{x^{3}} c .
\end{gathered}
$$

It is interesting to note that all points with fixed coordinate $\chi$ begin (at the initial instant of the expansion) to move away from each other at an infinite speed (according to (37), $\dot{a}=\dot{x} / x_{0}=$ $\left.\left(H_{\Lambda} / x_{0}\right) \sqrt{1+\beta x+x^{4}} / x\right)$. The geometric horizon begins to expand with velocity $2 c$, as does the Hubble distance, but the evolution of their velocities is opposite (see formulas (53) and (67)). For small $x$, the velocity $\dot{l}_{\mathrm{GHor}}$ grows very fast, while the velocity $\dot{l}_{\mathrm{H}}$ rapidly decreases, so that at $x=0.03$ they become equal to $2.50 \mathrm{c}$ and $1.56 \mathrm{c}$, respectively. The second horizon begins the expansion, as do all ordinary points of space, with an infinite speed, which decreases very rapidly.

Accelerations have similar evolution:

$$
\begin{gathered}
\ddot{l}_{\mathrm{GHor}}=l_{\mathrm{H}}^{0}(\ddot{a} \eta+2 \dot{a} \dot{\eta}+a \ddot{\eta})=\frac{\ddot{a}}{a} l_{\mathrm{GHor}}+l_{\mathrm{H}}^{0}\left(2 \dot{a} \frac{H_{0}}{a}-a \frac{H_{0}}{a^{2}} \dot{a}\right)=-\frac{4 \pi G}{3} \rho_{\mathrm{g}} l_{\mathrm{GHor}}+c H= \\
=c H_{\Lambda}\left[\frac{\sqrt{1+\beta x+x^{4}}}{x^{2}}+\frac{x^{4}-\beta x / 2-1}{x^{3}} I_{0}(x, \beta)\right] \sim c H_{\Lambda} I_{0}(\infty, \beta) x, \\
\ddot{l}_{\mathrm{KHor}}=l_{\mathrm{H}}^{0}\left[\ddot{a}\left(\eta_{\infty}-\eta\right)-2 \dot{a} \dot{\eta}-a \ddot{\eta}\right]=\frac{\ddot{a}}{a} l_{\mathrm{KHor}}-l_{\mathrm{H}}^{0}\left(2 \dot{a} \frac{H_{0}}{a}-a \frac{H_{0}}{a^{2}} \dot{a}\right)= \\
=-\frac{4 \pi G}{3} \rho_{\mathrm{g}} l_{\mathrm{H}}^{0} \frac{x}{x_{0}} \frac{I_{0}(\infty, \beta)-I_{0}(x, \beta)}{\left(\Omega_{\mathrm{r}}^{0} \Omega_{\Lambda}^{0}\right)^{1 / 4}-c H=} \\
=c H_{\Lambda}\left[\frac{x^{4}-\beta x / 2-1}{x^{3}}\left[I_{0}(\infty, \beta)-I_{0}(x, \beta)\right]-\frac{\sqrt{1+\beta x+x^{4}}}{x^{2}}\right] \sim-\frac{\beta}{8} \frac{\beta}{x^{3}} c H_{\Lambda} .
\end{gathered}
$$

At $x=x_{0}$, we obtain the current values of the velocities (see above) and accelerations: $\ddot{l}_{\mathrm{GH}}^{0}=$ $3.45 H_{\Lambda} c=19.9 \cdot 10^{-8} \mathrm{~cm} / \mathrm{s}^{2}, \ddot{l}_{\mathrm{KHor}}^{0}=-1.95 H_{\Lambda} c=-11.3 \cdot 10^{-8} \mathrm{~cm} / \mathrm{s}^{2}$. 

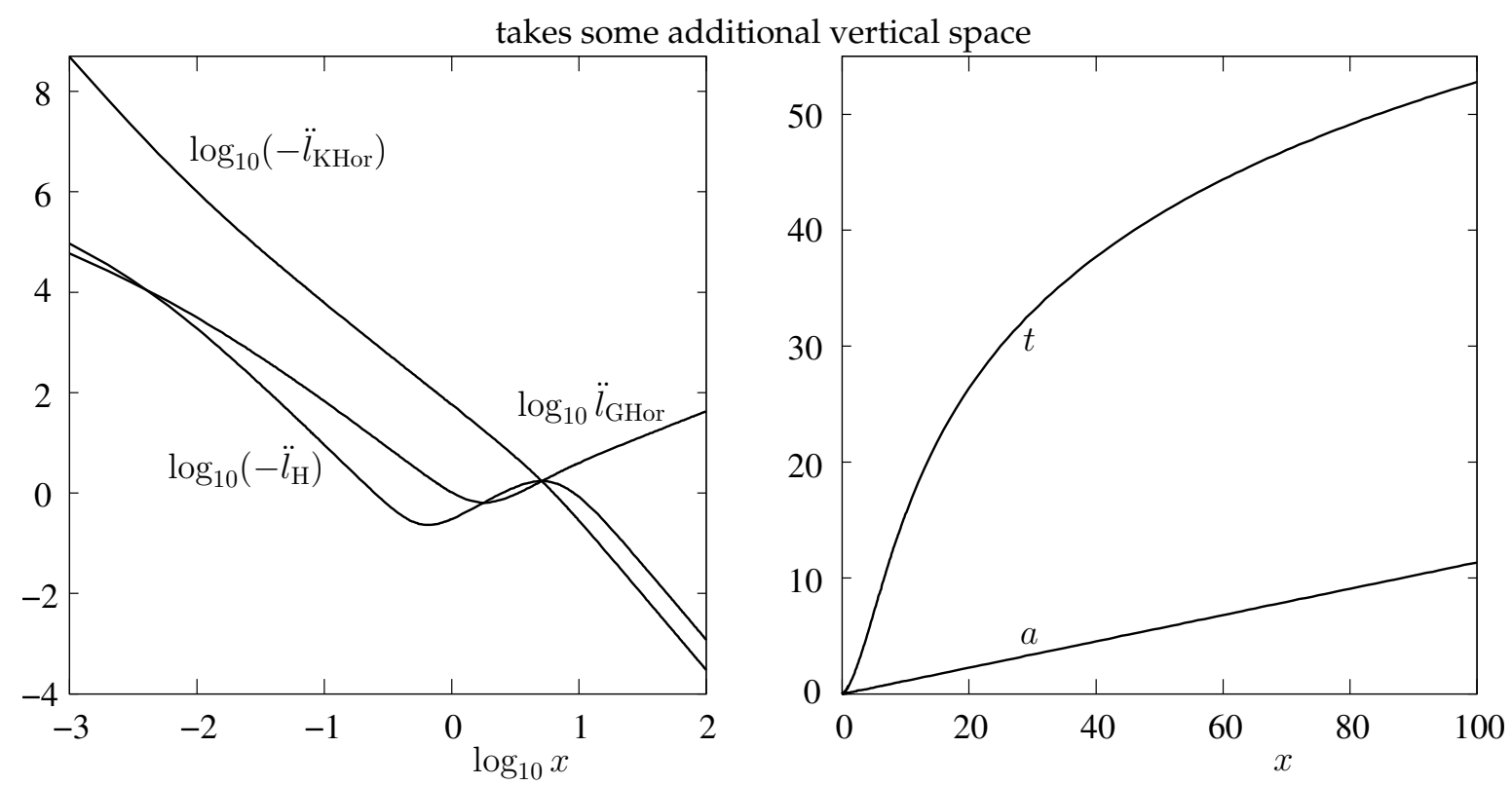

Figure 7. Left: Accelerations of the horizons and the Hubble distance. Right: Values of $a$ and $t$ as functions of $x$.

The speed of the first horizon increases, while that of the second horizon decreases. During the entire period of action of the cosmological acceleration $\left(6.5 \cdot 10^{9}\right.$ years $)$, the velocity of the first horizon increased from $3.31 c$ (by the value of $\eta$ for $\rho_{\mathrm{g}}=0$ in Table 1) to the current velocity of $4.32 c$, and the speed of the second horizon decreased from $0.479 c$ to $0.129 c$.

Figure 6, left presents the distances to the horizons, and Figure 6, right shows their velocities as a function of the parameter $x$. The figures give the same values for the Hubble distance. All distances are given in Gpc, and velocities are indicated in units of the speed of light. At first, until $\eta_{\infty}-\eta>\eta$, the distance to the second horizon is greater than that to the first horizon. The horizons intersected when $\eta_{\mathrm{crs}}=\eta_{\infty} / 2=2.23, x_{\mathrm{crs}}=4.08, z_{\mathrm{crs}}=1.677$ at an epoch $t_{\mathrm{crs}}=3.93$ billion years from the beginning, that is $t_{0}-t_{\text {crs }}=9.80$ billion years ago (earlier than the acceleration began), when the distance to the horizons was $3.58 \mathrm{Gpc}$. Prior to this, the first horizon determined the initial possibility to make observations (if there were observers at that time). Since then, the second horizon has become closer. Note, however, that the horizon effects differ. The first horizon (in fact, not it, but the physical horizon) limits the spherical region of space in which one can observe the past history of the universe, while the second defines those areas of information that will never reach the observer.

Figure 7, left plots the accelerations of the horizons and Hubble distance, measured in units of $c H_{\Lambda}$, on a logarithmic scale. Only the acceleration of the second horizon is a monotonic function; $\ddot{l}_{\mathrm{GHor}}$ has a minimum, while $\ddot{l}_{\mathrm{H}}$ has both a minimum and a maximum. The figures show a linear increase of the acceleration $\ddot{l}_{\mathrm{GH}}$ with $x$, and the equality of the rates of decrease of the accelerations $\ddot{l}_{\mathrm{H}}$ and $\ddot{l}$ according to the asymptotes (55) and (72). Figure 7, right shows the relationship of the scale factor $a$ and cosmological time $t$ with the parameter $x$.

\subsection{Connection with extraterrestrial civilizations}

Suppose that at the current epoch $\left(t=t_{0}, \eta=\eta_{0}\right)$ humans emit a radio signal in some direction. The distance to it increases; for a value of the time coordinate $\eta$ the distance will be equal to $l_{\mathrm{ph}}=$ $l_{\mathrm{H}}^{0} a(\eta)\left(\eta-\eta_{0}\right), \eta \geq \eta_{0}$. Its speed includes both the speed of expansion and the speed of light:

$$
\dot{l}_{\mathrm{ph}}=l_{\mathrm{H}}^{0} \dot{a}\left(\eta-\eta_{0}\right)+l_{\mathrm{H}}^{0} a \dot{\eta}=H l_{\mathrm{ph}}+c .
$$


Table 5. Objects along the signal path.

\begin{tabular}{|c|c|c|c|c|c|}
\hline$\eta_{\mathrm{O}}$ & $x_{\mathrm{O}}$ & $a_{\mathrm{O}}$ & $z_{\mathrm{O}}$ & $l_{\mathrm{O}}^{0} \mathrm{Gps}$ & $t_{\mathrm{O}}$ \\
\hline 1.99 & 2.67 & 0.303 & 2.30 & 5.69 & 2.89 \\
2.19 & 3.23 & 0.367 & 1.73 & 4.84 & 3.83 \\
2.39 & 3.87 & 0.440 & 1.28 & 3.98 & 4.95 \\
2.59 & 4.60 & 0.523 & 0.913 & 3.12 & 6.29 \\
2.79 & 5.46 & 0.620 & 0.613 & 2.27 & 7.88 \\
2.99 & 6.49 & 0.737 & 0.357 & 1.41 & 9.77 \\
\hline
\end{tabular}
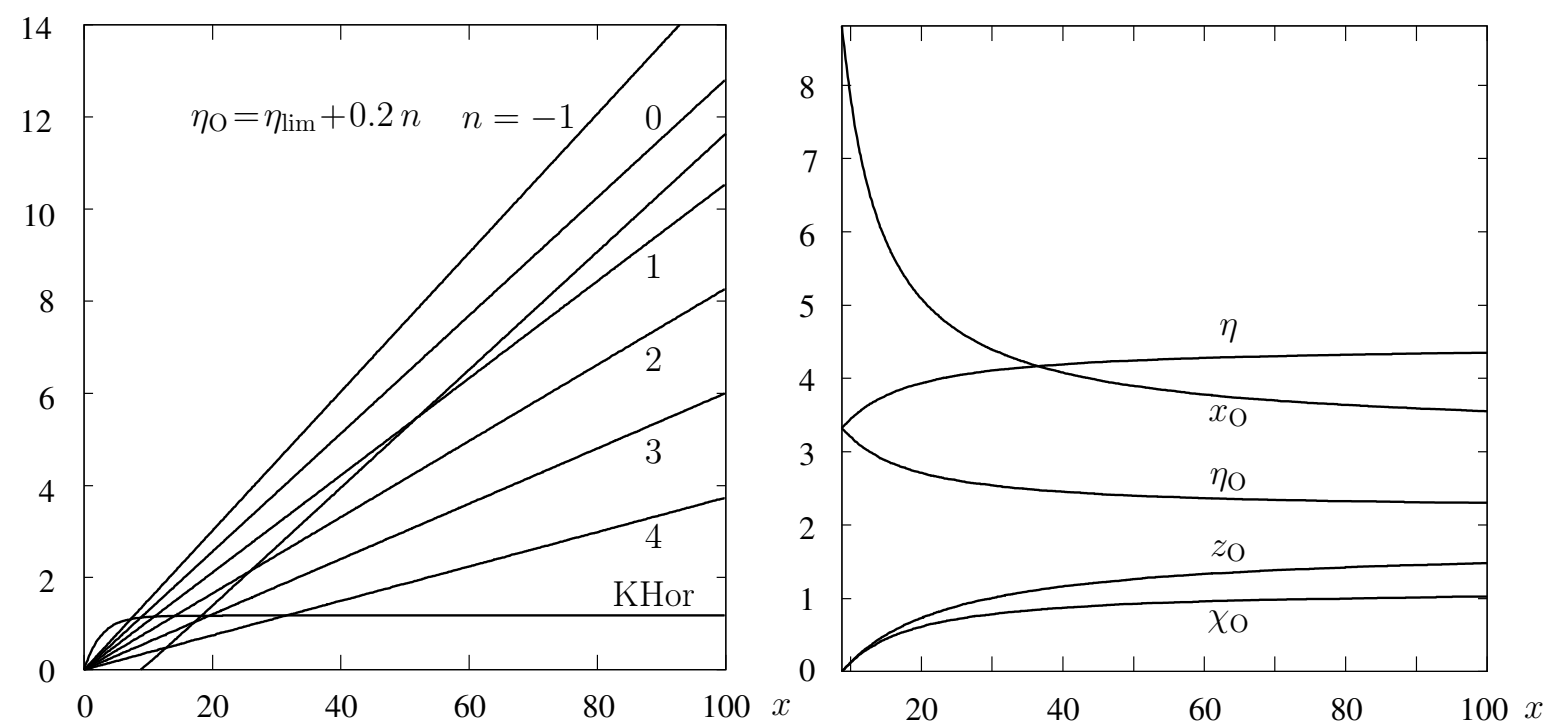

Figure 8. Left: Path of the signal to objects. Right: Objects reachable by the signal.

For brevity, we omit the factor $l_{\mathrm{H}}^{0}$, which means that we use distances measured in units of the modern Hubble distance. On the way, the signal passes by objects with fixed spatial coordinates $\chi_{\mathrm{O}}=\eta_{0}-\eta_{\mathrm{O}}$. Distances to these objects grow only due to the cosmological expansion, that is, increasing scale factor: $\tilde{l}_{\mathrm{O}}=a(\eta)\left(\eta_{0}-\eta_{\mathrm{O}}\right)$. The signal catches up with these objects when their distances from us become equal, which occurs at the moment $\eta_{\mathrm{mt}}$, when $\eta_{\mathrm{mt}}-\eta_{0}=\eta_{0}-\eta_{\mathrm{O}}, \eta_{\mathrm{mt}}=2 \eta_{0}-\eta_{\mathrm{O}}$; therefore, $\tilde{l}_{\mathrm{ph}}=\tilde{l}_{\mathrm{O}}=a\left(2 \eta_{0}-\eta_{\mathrm{O}}\right)\left(\eta_{0}-\eta_{\mathrm{O}}\right)$. Since $\eta_{\mathrm{mt}}$ cannot exceed $\eta_{\infty}$, the signal can meet for a finite (although, perhaps, very large) time only those objects whose coordinate satisfies $\chi_{\mathrm{O}}<\eta_{\infty}-\eta_{0}=1.13$. This implies that the coordinate has the same boundary as a photon traveling toward us. This boundary is the second horizon (see above), and $\eta_{\mathrm{O}}>\eta_{\lim }=2 \eta_{0}-\eta_{\infty}=2.19$.

Figure 8, left contains lines that plot the dependence of the distance $l_{\mathrm{O}}$ on the coordinate $x$ up to six objects. Positions of the signal path and the kinematic horizon are indicated as well. The objects are characterized by values of $\eta_{\mathrm{O}}=\eta_{\lim }+0.2 n, n=-1(1) 4$. The corresponding values of $x_{\mathrm{O}}$, the scale factor $a_{\mathrm{O}}=a\left(\eta_{\mathrm{O}}\right)$, and the redshift $z_{\mathrm{O}}$, as well as the current distances to these objects $l_{\mathrm{O}}^{0}$, are given in Table 5. It can be seen from the figure that the emitted signal reaches the objects only when $n=1,2,3,4$. The signal comes earlier to objects with larger values of $\eta_{\mathrm{O}}$, and hence smaller values of $z_{\mathrm{O}}$ and $l_{\mathrm{O}}$. These objects are located closer to the position of the signal output at the moment of its emission. The signal only catches up to objects with $n=3$ and 4 , for which the values of $\eta_{\mathrm{O}}$ are equal to 2.79 and 2.99, respectively, before they cross the second horizon. The condition of this is $\eta_{0}-\eta_{\mathrm{O}}<\eta_{\infty}-2 \eta_{\mathrm{O}}+\eta_{\mathrm{O}}$, that is, $\eta_{\mathrm{O}}>\eta_{\mathrm{h}}=\frac{3 \eta_{0}-\eta_{\infty}}{2}=2.758$. The distance between the object, 
which is now almost on the second horizon (the current distance to it is $4.84 \mathrm{Gpc}$ and its redshift is 1.72), and the signal for $\tilde{l}_{\mathrm{O}}^{0}=\eta_{\infty}-\eta_{0}-\varepsilon, \eta_{\mathrm{O}}=\eta_{\lim }+\varepsilon$ is

$$
l_{\mathrm{O}}-l_{\mathrm{ph}}=l_{\mathrm{H}}^{0} a(\eta)\left(\eta_{0}-\eta_{\mathrm{lim}}-\varepsilon\right)-l_{\mathrm{H}}^{0} a(\eta)\left(\eta-\eta_{0}\right)=l_{\mathrm{H}}^{0} a(\eta)\left(\eta_{\infty}-\varepsilon-\eta\right) \sim \frac{c}{H_{\Lambda}} \frac{\eta_{\infty}-\varepsilon-\eta}{\eta_{\infty}-\eta}
$$

since, according to eqs. (38) and (41), $\eta \sim \eta_{\infty}-\eta_{*} / x, a(\eta)=x / x_{0} \sim 1 /\left[\sqrt{\Omega_{\Lambda}^{0}}\left(\eta_{\infty}-\eta\right)\right]$ for $x \rightarrow \infty$, $\eta_{\infty}-\eta \ll 1$. The difference (74) tends to zero for $\eta \rightarrow \eta_{\infty}-\varepsilon$ if $\varepsilon>0$. Thus, in agreement with Figure 8 , left, the signal will still reach a given object if the object is located at least slightly closer than the second horizon. The time that the signal needs to meet the object is $t \sim \ln (1 / \varepsilon)$. If the object is located on the horizon $(\varepsilon=0)$, there remains an insurmountable distance $c / H_{\Lambda}=5.05 \mathrm{Gpc}$. The distance between the signal emitted now and objects currently located behind the second horizon will only increase with time. In addition, it will increase asymptotically as an exponential function. These objects are carried away by the exponential expansion, that is, by the repulsion of the dark energy. In models without repulsion the second horizon does not appear.

Figure 8 , right shows the dependences of the coordinates $x_{\mathrm{O}}, \eta_{\mathrm{O}}$ and $\chi_{\mathrm{O}}=\eta_{0}-\eta_{\mathrm{O}}$, as well as redshifts $z_{\mathrm{O}}$ of objects that the signal will reach at the time corresponding to its coordinate $x$. The figure plots also the dependence of the time coordinate $\eta$ on $x$. The signal emitted now will reach the second horizon when

$$
l_{\mathrm{ph}}=l_{\mathrm{H}}^{0} a\left(\eta_{\mathrm{h}}\right)\left(\eta_{\mathrm{h}}-\eta_{0}\right)=l_{\mathrm{KHor}}=l_{\mathrm{H}}^{0} a\left(\eta_{\mathrm{h}}\right)\left(\eta_{\infty}-\eta_{\mathrm{h}}\right), \quad \eta_{\mathrm{h}}=\frac{\eta_{0}+\eta_{\infty}}{2}=3.89 .
$$

The corresponding values are $x_{\mathrm{h}}=18.3, l_{\mathrm{ph}}=l_{\mathrm{KHor}}=5.02 \mathrm{Gpc}$, and $t_{\mathrm{h}}=24.9 \mathrm{Gyr}$. At this time an object with initial coordinate $x_{\mathrm{O}}=5.30$ will approach the horizon. This coordinate corresponds to $a_{\mathrm{O}}=0.601, z_{\mathrm{O}}=0.663$, and an initial distance to us of $l_{\mathrm{O}}^{0}=l_{\mathrm{H}}^{0}\left(\eta_{0}-\eta_{\mathrm{O}}\right)=1.45 \mathrm{Gpc}$, because if $l_{\mathrm{O}}=l_{\mathrm{ph}}=l_{\mathrm{KHor}}$ then $\eta_{0}-\eta_{\mathrm{O}}=\eta_{\mathrm{h}}-\eta_{0}=\eta_{\infty}-\eta_{\mathrm{h}}$ and $\eta_{\mathrm{O}}=\left(3 \eta_{0}-\eta_{\infty}\right) / 2=2.76$.

The signal sent by us can reach distances only up to $\approx 5 \mathrm{Gpc}$ to have any hope to get a reply. Exponential expansion of space entrains radiation both going away from us and directed toward us. Nevertheless, $5 \mathrm{Gpc}$ is a very large distance, and inside the sphere of such a radius there are many galaxies. If the signal hits a planet populated by intelligent creatures who have reached an advanced stage of civilization, they can receive it, understand, determine the direction from what it came, and reply. Then their signal will approach that place where humans were when we sent the first signal. The distance of their signal to us will change according to the formula $\tilde{l}_{\text {ret }}=a(\eta)\left(\eta_{\mathrm{mt}}-\eta_{0}-\eta\right)=$ $a(\eta)\left(\eta_{0}-\eta_{\mathrm{O}}-\eta\right)=a(\eta)\left(3 \eta_{0}-2 \eta_{\mathrm{O}}-\eta\right)$. Any reply will arrive at Earth at time $\eta_{\text {ret }}=3 \eta_{0}-2 \eta_{\mathrm{O}}$. If we want a reply to arrive at time $\eta_{\text {ret }}^{0}, \eta_{0}<\eta_{\text {ret }}^{0}<\eta_{\infty}$, then such a civilization should have $\eta_{\mathrm{O}}>\left(3 \eta_{0}-\eta_{\mathrm{ret}}^{0}\right) / 2$ and $\chi_{\mathrm{O}}<\left(\eta_{\mathrm{ret}}^{0}-\eta_{0}\right) / 2$. In an extreme case, if we assume that $\eta_{\mathrm{ret}}^{0}=\eta_{\infty}$, then the condition $\eta_{\text {ret }}<\eta_{\infty}$ imposes a restriction on the coordinate $\eta_{0}: \eta_{O}>\left(3 \eta_{0}-\eta_{\infty}\right) / 2$. This restriction coincides with the condition that the signal reaches the object (civilization) before the latter reaches the second horizon. The restriction on the spatial coordinate is as it was previously: $\chi_{O}=\eta_{0}-\eta_{O}<\left(\eta_{\infty}-\eta_{0}\right) / 2$.

It is clear that it makes sense to send a signal to objects located closer than several dozen light years, otherwise any possible reply would take too long. Undoubtedly, it will be necessary to limit the search within our Galaxy and even the immediate vicinity of the solar system. Even in this case the signals must either be sent in a very narrow cone, or they should be sufficiently energetic so that they can be received at a greater distance.

Although the above arguments have a purely theoretical or even academic character, they establish restrictions on the limits imposed by the model. They can be related either to epochs when our civilization on the Earth has not existed yet, or has not been able to realize connections with other civilizations, or to the epochs when the Sun and Earth will no longer exist in their current form. 
However, these same arguments apply to any arbitrary location in the universe and to civilizations that may arise and prosper at any time.

\section{Conclusion}

In this paper we have summarized results of the Standard model that reveal some of its quantitative properties. After a brief excursion into the history of creation of cosmological models, we have presented the two Friedmann-Lemaitre equations, which describe a uniform and isotropic universe, and have restated the definitions of the critical values and five cosmological distances. Based on the compatibility condition of two equations and on the equations of state, we have derived the laws of the change with time of the mass density of four noninteracting components: the density of matter decreases as the third order of the scale factor and the density of radiation and neutrinos as the fourth order, while the dark energy density is unchanged. These laws provide solutions of the cosmological equations in quadratures.

The equations are specified for the flat space-time model. Using the parameters of this model obtained from observations: Hubble constant, $H_{0}=70 \mathrm{~km} / \mathrm{s} / \mathrm{Mpc}$, temperature of the cosmic background radiation, $T_{0}=2.7727 \mathrm{~K}$, dark energy fraction of the total cosmological mass density, $\Omega_{\Lambda}^{0}=0.72$, we have determined the current Hubble distance $l_{\mathrm{H}}^{0}=4.28 \mathrm{Gpc}$, the critical density $\rho_{\mathrm{c}}=9.2 \cdot 10^{-30} \mathrm{~g} / \mathrm{cm}^{3}$, and the fractional contributions to $\rho_{\mathrm{c}}$ of the radiation, $5 \cdot 10^{-5}$, six types of neutrinos $6.9 \cdot 10^{-5}$, and dust-like matter, $\approx 0.28$, which includes dark matter. They define the relationships of the scale factor $a=1 /(1+z)$ (and, thus, of the redshift $z$ ), the conformal dimensionless time coordinate $\eta$, and the dimensionless parameter $x$ with cosmological time $t$. For the early and late stages of expansion, simple and sufficiently accurate approximations of these relationships have been obtained. We have shown that, in contrast to redshift, coordinates $\eta$ and $x$ are not tied to a specific epoch of evolution. Several dimensionless parameters of the model have been introduced that are also free from such binding, and their quantitative values have been derived.

It has been shown that major events in the evolution of the universe occurred near the beginning, when the dominant carrier of the mass density transferred from radiation and neutrinos to "dust" corresponding to $z$ changing from 5500 to 1000 - and then later when dark energy became dominant, at $z=0.7$ to 0.4 .

Dependencies of different types of distances have been calculated as functions of parameters $x$ and $z$, as well as speeds of their changes. A difference between the cosmological redshift and classical Doppler effect was stressed, which was explained by the fact that a shift of the frequency of a photon occurs not only at the time of its emitting by a cosmical object, but at every point of its path to the observer.

We have discussed the concepts of two horizons: geometric, inherent in any expanding model, and kinematic, typical for models expanding with an acceleration. Distances to these horizons, along with speeds and accelerations of evolution of these distances, have been derived as functions of time. Current distances to the horizons are $14.2 \mathrm{Gpc}$ for the first horizon and $4.84 \mathrm{Gpc}$ to the second horizon. The horizons crossed each other $9.8 \mathrm{Gyr}$ ago, when the distance to them was $3.58 \mathrm{Gpc}$.

The current acceleration of expanding space represents the most surprising value: at the Hubble distance, where the expansion rate is equal to the speed of light, the acceleration is about $4 \AA / \mathrm{s}^{2}$. Such a value of the acceleration has been reached over the past 6.5 billion years, while it was zero at the beginning of the expansion. A limit on the acceleration for $t \rightarrow \infty$ at the limit of the Hubble distance is $5.7 \mathrm{~A} / \mathrm{s}^{2}$. Even at the current horizon the acceleration is only slightly higher, $\approx 20 \mathrm{~A} / \mathrm{s}^{2}$. However, tens of billions of years into the future, the acceleration of the horizon and scale factor (indeed, all scales) will increase over time exponentially with an exponent of $t / t_{\Lambda}$, where $t_{\Lambda}=16.5$ Gyrs. This means that a second inflation will occur.

We have estimated the rate of change of redshifts and apparent luminosities of objects with increasing age of the universe. For distant objects with $z>13.2$, the apparent luminosity can grow 
with time. However, detection of these effects requires very long time intervals between observations, as well as significant improvements in the capabilities of observational instruments in the future.

We have estimated distances across which our signal emitted from the Earth can reach extraterrestrial civilizations and from which they can respond to us. These distances are quite large, on the order of $5 \mathrm{Gpc}$, so that they do not limit the possibilities of contact with other civilizations in the universe.

The above discussion provides a quantitative description of various geometric and kinematic properties of the Standard model that is currently considered to be an accurate description of the universe. Future observationally driven revisions of the cosmological parameters such as the Hubble constant would require updates to the exact values of the parameters that we have derived. Nevertheless, as long as the cosmological constant dominates the current and future energy of the universe, seemingly odd features, such as the presence of two horizons, will remain intact.

Acknowledgments: SJ acknowledges partial support from Russian Science Foundation grant 17-12-01029.

Author Contributions: DN and SJ contributed 70\% and 30\%, respectively, to this work.

Conflicts of Interest: The authors declare no conflict of interest.

\section{References}

1. Zeldovich, Ya.B.; Novikov, I.D. 1975. The Structure and Evolution of the Universe. University of Chicago Press, Chicago. 1983.

2. Narlikar, J.V. Introduction to Cosmology. Cambridge, Cambridge University Press. 1993.

3. Misner, T.W.; Thorn, K.S.; Wheeler, J.A. Gravitation. San Francisco, Freeman. 1972.

4. Weinberg, S. Gravitation and Cosmology: Principles and Applications of the General Theory of Relativity. New York, John Wiley and Sons, Inc. 1972.

5. Gorbunov, D.C.; Rubakov, V.A. Introduction to the Theory of the Early Universe. The Theory of Hot Big Bang. M., URSS. 2008.

6. Einstein, A. Die Grundlage der allgemeine Relativistätstheorie. Ann. d. Phys. 1916, 49, 760.

7. Einstein, A. Kosmologische Betrachtugen zur allgemainen Relativitatstheories. Sitsungsberichte der Preuss. Acad. Wiss. 1917, 142-152. (English translation: H.A.Lorents, A.Einstein, H.Minkowski, H.Weil. 1950. The principle of relativity. 177-188. Methuen, London.)

8. Eddington, A. The Mathematical Theory of Relativity. Second edition. Cambridge. At the University Press. 1924.

9. de Sitter, W. On Einstein's theory of gravitation and its astronomical consequencies, Third paper. Monthly Notices Roy. Astron. Soc. 1917, 78, 3-28.

10. Friedmann, A. Über die Krümmung des Raumes. Zeitschrifts für Physik. 1922, 10, 377-386.

11. Friedmann, A. Über die Möglichkeit einer Welt mit konstanter negativer Krümmung des Raumes. Zeitschrifts für Physik, 1924, 21, 326.

12. Lemaître, G. Un universe homogéne de masse constante et de rayion croissante rendant compte de la vitesse radiale des nébuleses extragalactiques. Annales de la Société scientifique de Bruxelles. 1927, $47 \mathrm{~A}$, 41. A homogeneous universe of constant mass and increasing radius accounting for the radial velocity of extra-galactic nebulae. Mon. Not. R. Astron. Soc. 1931, 91, 483-490.

13. Lemaître, G. The expanding universe. Mon. Not. R. Astron. Soc. 1931, 91, 490-501.

14. Einstein, A. Grundgedanken und Probleme der Relativitátsthedorie. In "Nobelstiftelsen, Les Prix Nobel en 1921-1922". Impremerie Royal, Stockholm. 1923.

15. Einstein, A. Zum kosmologischen Problem der allgemainen Relativitätstheorie. Sitzungsber. Preuss. Acad. Wiss., phys.-math. K1., 1931, 235-237.

16. Hubble, E. A relation between distance and radial velocity among extragalactic nebulae. Proc. Nat. Acad. Sci. USA, 1929, 15, 168.

17. Sandage, A. Observational tests of world models. Ann. Rev. Astron. Astrophys. 1988, 26, 561-630.

18. Sandage, A. Current problems in the extragalactic distance scale. Astrophys. J. 1958, 127, 513-527.

19. Sandage, A.; Tammann, G.A. Steps towards the Hubble constant. VIII. The global value. Astrophys. J. 1982, 256, 339-345. 
20. Sandage, A. The redshift-distance relation. II. The Hubble diagram and its scatter for first-ranked cluster galaxies: a formal value for $q_{0}$. Astrophys. J. 1972, 178, 1-24.

21. Hoyle, F.; Burbidge, G.; Narlikar, J.V. A Different Approach to Cosmology. From a static universe through the big bang towards reality. Cambridge University Press. 2000.

22. Frieman, J.A.; Turner, M.S.; Huterer, D. Dark energy and accelerating universe. Annu. Rev. Astron. Astrophys. 2008, 46, 385-432.

23. Gliner, E.B. Algebraic properties of the energy-momentum tensor and vacuum-like states of matter. Zhurn. Experim. Theor. Fizik. 1965, 49, 542-548.

24. Guth, A. Inflationary universe: a possible solution to the horizon and flatness problems. Phys. Rev. D. 1981, 23, 347-356.

25. Linde, A.D. The physics of elementary particles and inflationary cosmology. M. Nauka. 1990.

26. Riess, A.S.; et al. Observational evidence from supernovae for an accelerating universe and a cosmological constant. Astron. J. 1998, 116, 1009-1038.

27. Perlmuter, S.; et al. Measurements of $\Omega$ and $\Lambda$ from 42 high-redshift supernovae. Astrophys. J. 1999, 517, 565-586.

28. Knop, R.A.; Aldering, G.; Amanullah, R.; Astier, P.; Blanc, G.; et al. New constraints on $\Omega_{M}, \Omega_{\Lambda}$, and $w$ from an independent set of 11 high-redshift supernovae observed with the Hubble Space Telescope. Astrophys. J. 2003, 598, 102-137.

29. Hinshaw, G.; Larson, D.; Komatsu, E.; et al. Nine-year Wilkinson Microwave Anisotropy Probe (WMAP) observations: cosmological parameters results. Astrophys. J. Suppl. Series. 2013, 208, 19.

30. DES Collaboration: Abbott, T.M.C.; Allam, S.; Andersen, P.; et al. First Cosmology Results using Type Ia Supernovae from the Dark Energy Survey: Constraints on Cosmological Parameters. arXiv:astro-ph/1811.02374.

31. Macaulay, E. R.; Nichol, C.; Bacon, D.; et al. DES Collaboration. First Cosmological Results using Type Ia Supernovae from the Dark Energy Survey: Measurement of the Hubble Constant. arXiv:astro-ph/1811.02376.

32. Nagirner, D. I.; Turichina, D. G. The effect of neutrino mass in cosmology. Astrophysics, 2019, 62, 108-128.

33. McCrea, W.H. Observable relations in relativistic cosmology. Zeitschrift für Astrophysik. 1935, 9, $290-314$.

34. Harrison, E. The redshift-distance and velocity-distance laws. Astrophys. J. 1993, 403, $28-31$.

35. Alpher, R.; Herman, R. The Origin and Abundance Distribution of the Elements. Ann. Rev. Nucl. Astropart. Sci. 1953, 2, 1-40.

36. Sandage, A. The change of redshift and apparent luminosity of galaxies due to the deceleration of selected expanding universes. Astrophys. J. 1962, 136, 319-333.

37. McVittie, G.C. Appendix. Astrophys. J. 1962, 136, 334-338.

38. Loeb, A. Direct measurement of cosmological parameters from the cosmic deceleration of extragalactic objects. Astrophys. J. 1998, 499, L111-L114.

39. Liske, J.; et al. Cosmic dynamics in the era of Extremely Large Telescopes. Mon. Not. R. Astron. Soc. 2008, $386,1192-1218$.

40. Rindler, W. Visual horizons in world-models. Mon. Not. R. Astron. Soc. 1956, 116, 662-677.

41. Margalef-Bentabol, B.; Margalef-Bentabol; J.; Cepa, J. Evolution of the cosmologycal horizons in a universe with coutably infinitely many state equations. Journal of Cosmology and Astroparticle Physics. 2013. 015. arXiv:astro-ph/1302.2186. 